\title{
Axion and hidden photon dark matter detection with multilayer optical haloscopes
}

\author{
Masha Baryakhtar, Junwu Huang, ${ }^{\dagger}$ and Robert Lasenby ${ }^{\ddagger}$ \\ Perimeter Institute for Theoretical Physics, 31 Caroline Street North, Waterloo, Ontario N2L 2Y5, Canada
}

(Received 15 May 2018; published 3 August 2018)

\begin{abstract}
A well-motivated class of dark matter candidates, including axions and dark photons, takes the form of coherent oscillations of a light bosonic field. If the dark matter couples to standard model states, it may be possible to detect it via absorptions in a laboratory target. Current experiments of this kind include cavitybased resonators that convert bosonic dark matter to electromagnetic fields, operating at microwave frequencies. We propose a new class of detectors at higher frequencies, from the infrared through the ultraviolet, based on the dielectric haloscope concept. In periodic photonic materials, bosonic dark matter can efficiently convert to detectable single photons. With feasible experimental techniques, these detectors can probe significant new parameter space for axion and dark photon dark matter in the $0.1-10 \mathrm{eV}$ mass range.
\end{abstract}

DOI: 10.1103/PhysRevD.98.035006

\section{INTRODUCTION}

There is overwhelming evidence that the majority of the matter density of the universe takes some beyond-standardmodel form, referred to as dark matter (DM) $[1,2]$. Despite this, the form of the dark matter remains almost entirely unknown. If, like standard model (SM) matter, it is a relic from the hot thermal plasma of the early universe, then the fact that it is "cold" (low-velocity) today means that it cannot consist of particles lighter than $\sim \mathrm{keV}[3,4]$. However, there are a range of alternative, nonthermal production mechanisms which could generate a viable cold dark population of lighter particles. In many beyond-the-standard-model theories, these light new particles can naturally have very small couplings to SM states, allowing them to be stable and hardto-detect. Thus, such particles can serve as attractive dark matter candidates. At masses $\lesssim 100 \mathrm{eV}$, the dark matter must be bosonic, since the Pauli exclusion principle forbids fermionic DM from having the dense, low-velocity distributions observed in galaxies [5].

Unless there is a symmetry preventing it, the leadingorder interaction between light bosonic dark matter and standard model matter will be absorption and emission of single DM particles. This is true for the simplest and most

\footnotetext{
*mbaryakhtar@perimeterinstitute.ca

jhuang@perimeterinstitute.ca

rlasenby@perimeterinstitute.ca
}

Published by the American Physical Society under the terms of the Creative Commons Attribution 4.0 International license. Further distribution of this work must maintain attribution to the author(s) and the published article's title, journal citation, and DOI. Funded by SCOAP ${ }^{3}$. attractive light DM models, such as axions [6-8] or dark photons [9]. Accordingly, a range of existing and proposed experiments aims to detect the absorption of light DM through different mechanisms (see [10-12] for reviews of axion and dark photon DM detection experiments). However, many of these are not sensitive to DM masses far above the microwave frequency range. In this paper, we discuss how to extend the search for light dark matter candidates to higher masses, from 0.1 to $10 \mathrm{eV}$.

For many kinds of dark matter couplings, DM to photon conversion is a promising experimental approach, transferring the entire rest mass energy of the dark matter to readily-detectable photons. At DM Compton wavelengths around or above meter scales, conversion experiments based on resonant receivers [13-15] are a practical solution, as illustrated by the ADMX experiment [16,17], and by a range of ongoing and proposed experiments at similar and lower frequencies [18-21]. At higher DM masses, filling a large volume with resonant elements, such as cavities matching the DM Compton wavelength, becomes difficult. Consequently, other forms of target structure that can correct the mismatch between the DM and photon dispersion relations are more practical. "Dielectric haloscopes" [22-24] provide an example of this idea; a periodic structure of alternating dielectrics modifies photon propagation in the target volume, enabling DM-to-photon conversion for DM Compton wavelengths matching the target periodicity. The proposed MADMAX experiment $[22,25,26]$ aims to search for axion DM using this technique, over a mass range $40-400 \mu \mathrm{eV}$.

The main topic of this paper will be extending the dielectric haloscope concept to higher-than-microwave frequencies. At these shorter wavelengths, it becomes more difficult to construct and manipulate individual, wavelength-scale 
elements. On the other hand, it is possible to make bulk materials whose optical properties vary on the relevant scales, all the way down to ultraviolet wavelengths. These "photonic" materials have been used to create many novel optical devices, such as very high quality cavities and filters [27-30]. The simplest and most widely used examples are multilayer films, as employed in optical coatings.

There are a number of reasons why higher-mass bosonic DM is an attractive target for experimental searches. Practically speaking, single-photon detection becomes significantly easier at energies $\gtrsim 0.1 \mathrm{eV}$, corresponding to the energy resolution of superconducting detectors, as made use of in [31]. On the theoretical side, there are ranges of parameter space where simple early-universe production mechanisms can produce the correct DM abundance, with couplings below current constraints; in particular, purely gravitational production during inflation can result in a DM abundance of light bosons. Such cold bosonic dark matter acts as a coherent classical-like field, oscillating at a frequency set by its mass $m$ and with an amplitude set by $m$ and the dark matter density. It is coherent over times of order $t_{\mathrm{coh}} \sim\left(m v^{2}\right)^{-1}$ and lengths of order $l_{\mathrm{coh}} \sim(m v)^{-1}$, where $v \sim 10^{-3}$ is the virial velocity in the galaxy.

In this work we outline an experimental proposal using multilayer films, combined with a sensitive photodetector, to search for bosonic dark matter. Alternating layers of

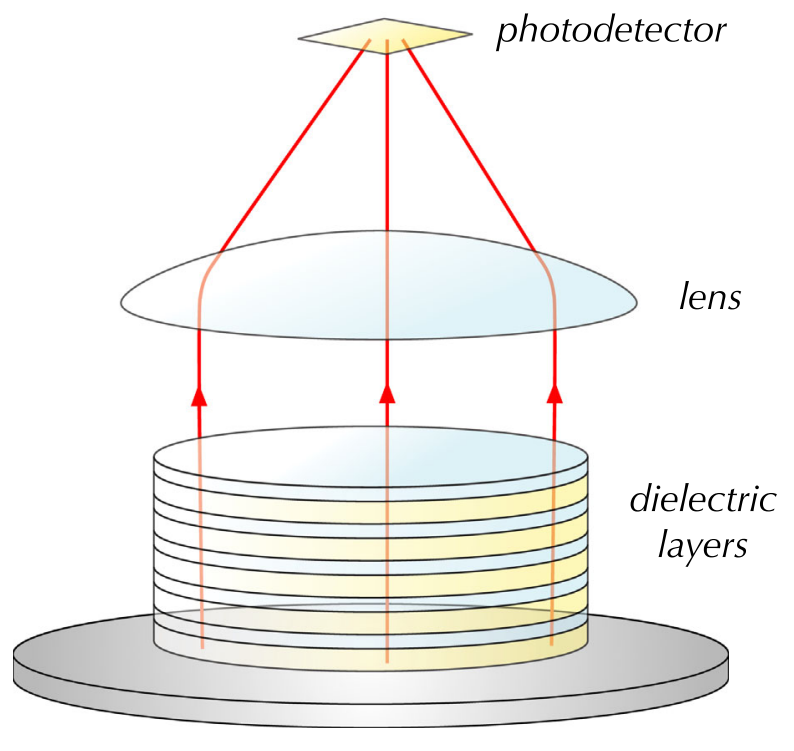

mirror

FIG. 1. Sketch of our proposed experimental setup. A stack of dielectric layers, with alternating indices of refraction, is placed on a mirror. In the presence of the right type of background DM oscillation (e.g., dark photon DM), at a frequency corresponding to the inverse spacing between the layers, the layers will emit photons in their normal direction (shown as red lines). These can be focused onto a sensitive, low-noise detector. To detect axion DM with a coupling to photons, a magnetic field should be applied parallel to the layers. commonly used dielectrics with different indices of refraction lead to coherent conversion of dark photon, and, in the presence of an applied magnetic field, axion, dark matter to photons. The resulting photons emerge in a direction perpendicular to the layers, and are focused onto a detector (Fig. 1). These setups have close-to-optimal DM absorption rates (in a DM mass-averaged sense). Small volumes $\left(\sim \mathrm{cm}^{3}\right)$ of layered material could achieve sensitivities several orders of magnitude better than existing constraints for these dark matter candidates.

Section II discusses the theory of DM absorption in layered materials; this can be skipped by readers more interested in experimental details. In Sec. III, we summarize these theoretical results, discuss concrete, illustrative examples of how such an experiment might be realized, and analyze the sensitivity of these setups. Section IV discusses sensitivity to other forms of DM. In Sec. V, we give a brief overview of DM production mechanisms, and conclude by discussing future extensions and comparing our proposal to other experiments in Sec. VI.

\section{MULTILAYER OPTICAL FILMS}

In this section, we will start by giving a brief overview of the physics of DM to photon conversion, in the simplest photonic materials: multilayer films. By the scaling properties of Maxwell's equations, this is a rescaled version of the physics of dielectric haloscopes at microwave frequencies, as derived in depth by various publications from the MADMAX collaboration [22,26]. Here, we derive the results needed for our experimental configurations from a slightly different perspective, giving some additional physical insight.

\section{A. Axion conversion in layered materials}

DM to photon conversion is an especially attractive experimental strategy for models in which the DM couples directly to the EM field-for example, an axion $a$ with Lagrangian

$$
\begin{aligned}
& \mathcal{L} \supset \frac{1}{2}\left(\partial_{\mu} a\right)^{2}-V(a)-\frac{1}{4} g_{a \gamma \gamma} a F_{\mu \nu} \tilde{F}^{\mu \nu} \\
& =\frac{1}{2}\left(\partial_{\mu} a\right)^{2}-V(a)+g_{a \gamma \gamma} a E \cdot B,
\end{aligned}
$$

where we take the $(+---)$ signature, and use the convention $\epsilon_{0123}=-1$. Except where indicated, we use natural units with $c=\hbar=1$. By "axion," we will mean a spin-0 particle with (dominantly) odd-parity couplings to SM states, of which a QCD axion would be a particular example. An axion generally has a periodic potential, $V(a) \simeq-m^{2} f_{a}^{2} \cos \left(a / f_{a}\right)$, where $f_{a}$ is the axion's "decay constant," and $m$ is its mass. The "natural" expectation for the coupling to photons is that $g_{a \gamma \gamma} \simeq \frac{\alpha_{\mathrm{EM}}}{2 \pi f_{a}}$, where $\alpha_{\mathrm{EM}}$ is the fine structure constant [32]. As discussed in Sec. V, a dark 
matter abundance of these axions could be produced via a number of mechanisms. We will take such an axion as our prototypical example for the rest of this section, commenting later on sensitivity to other DM candidates.

In the presence of the $a F \tilde{F}$ interaction term, the Maxwell equations are modified to [33]

$$
\begin{aligned}
\nabla \cdot E & =\rho-g \nabla a \cdot B, \\
\nabla \times B & =\partial_{t} E+J+g(\dot{a} B+\nabla a \times E), \\
\nabla \cdot B & =0, \quad \nabla \times E=-\partial_{t} B,
\end{aligned}
$$

where we abbreviate $g_{\text {ayr }}$ as $g$, here and following. In particular, a uniform oscillating $a$ field has the same effects as an oscillating current density $g \dot{a} B$ in the direction of $B$, while other contributions are suppressed by the DM velocity. Accordingly, the ideal "target" for axion to photon conversion is a strong magnetic field; in common with other axion detection experiments, we will use an approximately uniform field from a large magnet.

The remaining difficulty in accomplishing $a-\gamma$ conversion is achieving a setup in which the interaction does not cancel out when integrated over the target. For resonant cavity experiments such as ADMX, modes above the few lowest-lying ones will have small overlap with the effective current $g \dot{a} B$. In target materials with small-scale periodicity, another formulation is that DM-photon conversion process must conserve (pseudo)momentum, up to the material's reciprocal lattice vectors, analogously to Bragg scattering. As Fig. 2 schematically illustrates, this means that the target must have structure on scales set by the Compton wavelength of the DM.

A simple way to realize the appropriate structure is with photonic materials, which have spatially nonuniform optical properties. ${ }^{1}$ Figure 1 shows the schematic structure of such a detector using a 1D photonic material: a set of dielectric layers with alternating permittivities. As illustrated by Fig. 2, most of the momentum of the emitted photons "comes from" the periodicity of the material, so they are emitted in a tight cone around a particular angle. They can then be focused down onto a small, sensitive detector. This is precisely the experimental setup proposed, at microwave frequencies, by the MADMAX collaboration [22].

The simplest situations to analyze correspond to periodic layered structures. Photon modes in an infinitely extended periodic medium are Bloch modes; if the layers are uniform in the $x, y$ plane, then

$$
E(r)=e^{i k \cdot r} u_{k}(z)
$$

\footnotetext{
${ }^{1}$ Another possibility would be to use a spatially nonuniform magnetic field, as per the ORPHEUS experiment [34], but this is harder to implement with a multitesla field.
}

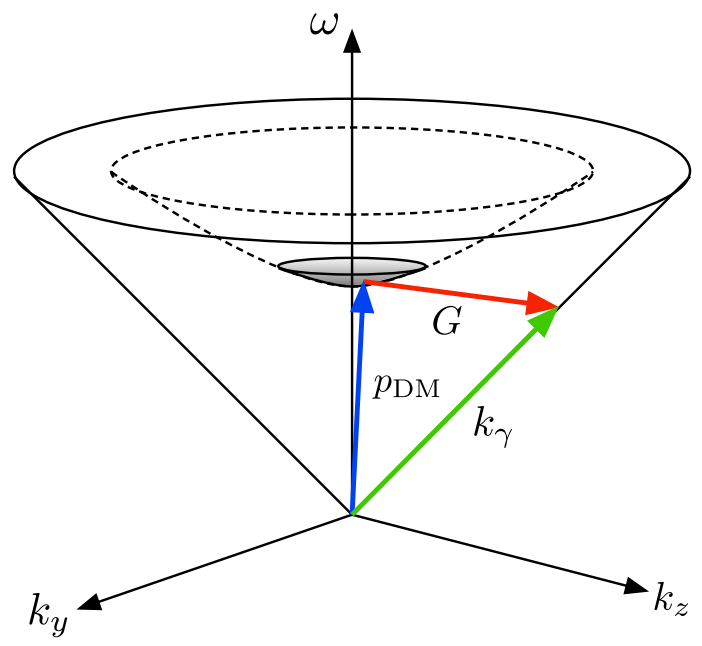

FIG. 2. Schematic illustration of DM with 4-momentum $p_{\text {DM }}$ being converted to a photon with momentum $k_{\gamma}$. The dotted line indicates the mass shell of the DM particle; since the DM is nonrelativistic, a given quantum will have small velocity, corresponding to the shaded region. For this illustration, we have assumed that photons have a linear dispersion in the target material (see Fig. 3 for how this can be modified). In periodic materials, conservation of pseudo-momentum inside the material requires that $k_{\gamma}-p_{\mathrm{DM}} \equiv G$ is a reciprocal lattice vector of the medium.

with $u_{k}(z+d)=u_{k}(z)$, where $d$ is the periodicity in $z$ of the material. Taking $k_{z}$ to lie within the first Brillouin zone, $(-\pi / d, \pi / d)$, we can label different modes at the same $k_{z}$ by band number. Figure 3 illustrates part of this band structure for some simple periodic materials. The modes in a large but finite stack will be similar to those in the infinite case. As noted above, the DM momentum is small compared to its mass, so we are interested the modes for which $k_{\perp}$ is small compared to $d^{-1}$. In particular, a DM mode with frequency $\omega$, and small momentum $k$, can convert to a photon of frequency $\omega$ if the Bloch mode at that frequency has Bloch momentum close enough to $k$.

The DM absorption rate of any 1D stack configuration can be calculated via transfer matrices, as presented in [26]. However, in terms of developing a physical picture, it can be useful to present the calculation, at least around the $k=0$ points, in terms of the unforced photon modes in the layers (this corresponds to the "overlap integral" calculations of $[22,26])$. The simplest-to-analyse periodic configurations have layers of alternating refractive indices $n_{1}$, $n_{2}$, with thicknesses $d_{1}, d_{2}$ such that their phase depths are equal, $n_{1} d_{1}=n_{2} d_{2}=\pi / \omega$.

We start by ignoring the DM velocity, and treating the DM field as a classical background oscillating at frequency $\omega$, with $a(t)=a_{0} \sin \omega t$ (treating the DM field as classical will always be a good approximation in the regimes we consider). This induces an electric field $E(x, t)$ in the material, resulting in an instantaneous absorbed power of 

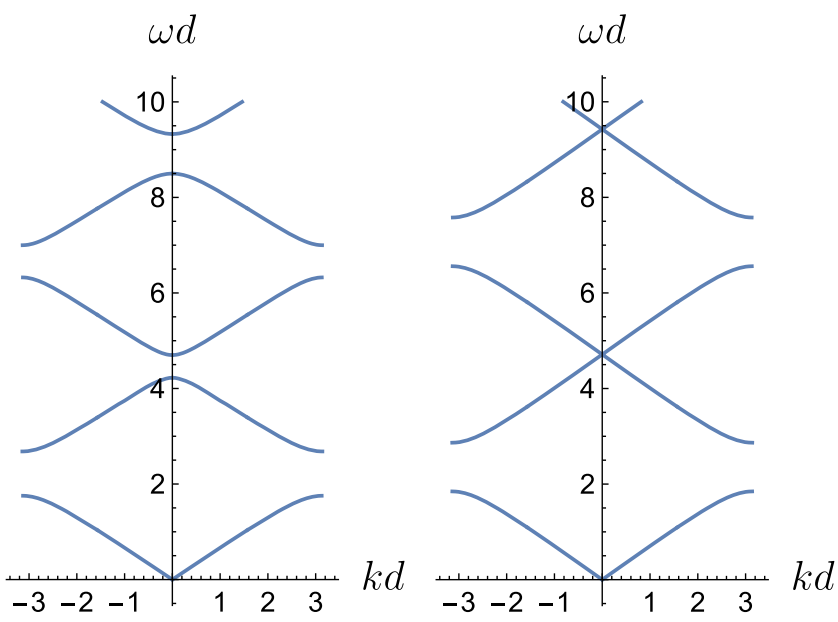

FIG. 3. Left-hand plot: dispersion relation for photon propagation in a periodic medium with alternating layers of refractive indices $n_{1}=1, n_{2}=2$, and widths $d_{1}=1, d_{2}=0.7$ (in arbitrary units), where $d=d_{1}+d_{2}$ is the periodicity. The momentum $k$ is taken to be in the same direction as the material periodicity. Right-hand plot: as for the left-hand plot, but with $d_{1}=1$, $d_{2}=0.5$. As discussed in Sec. II A, this configuration has no band-gaps around the $k=0$ points.

$$
P_{\mathrm{int}} \simeq \int d V E \cdot \partial_{t}\left(g a B_{0}\right)
$$

where $B_{0}$ is the background magnetic field (this is the instantaneous energy flow from the DM field to the SM target).

At points in the cycle when $a=0$, the electric field is continuous at the layer interfaces. The EM fields, at these moments, must correspond to those of a free photon mode. The out-of-phase component of the electric field does not contribute to the absorbed power, so we can calculate this taking only the free mode into account. For a half-wave stack with refractive indices $n_{1}, n_{2}$, and layer thicknesses $d_{i}=\pi /\left(n_{i} \omega\right)$, there is a mode with electric field (parallel to the layers)

$E= \begin{cases}\frac{1}{n_{1}} \sin \left(k_{1} z\right) E_{0} \cos \omega t & \left(0 \leq z \leq d_{1}\right) \\ \frac{1}{n_{2}} \sin \left(k_{2}\left(z-d_{1}\right)\right) E_{0} \cos \omega t & \left(d_{1} \leq z \leq d_{1}+d_{2}\right),\end{cases}$

as illustrated in Fig. 4 (there is also the out-of-phase mode, for which $E$ averages to zero across a period). If $E_{0}$ is the amplitude in this mode excited by the DM oscillation, then the instantaneous absorbed power is

$$
P_{\mathrm{int}} \simeq N A\left(\frac{1}{n_{1}^{2}}-\frac{1}{n_{2}^{2}}\right) E_{0} g a_{0} B_{0} \cos ^{2} \omega t
$$

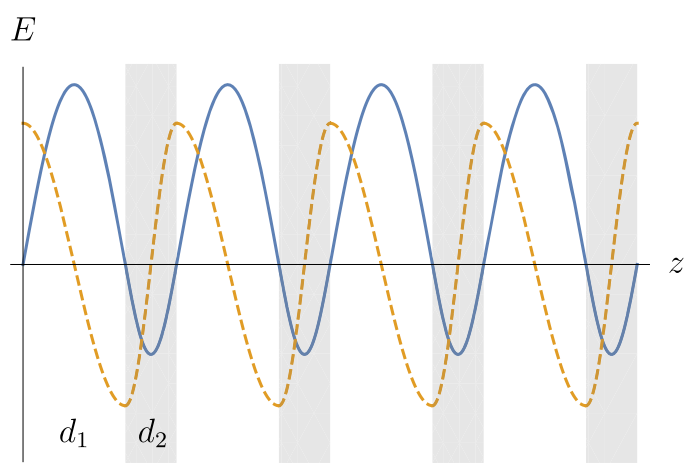

FIG. 4. Electric field for the second-band modes with Bloch momentum $k=0$, in infinite periodic alternating layers with refractive indices $n_{1}=1, n_{2}=2$, and thicknesses $d_{1}=1, d_{2}=$ 0.5 (as per the right-hand plot of Fig. 3). The $y$ axis denotes the amplitude of the electric field, which is transverse to the layers. The solid-line mode, which has nonzero $\int d z E$ over a period, is the one excited by a background DM field. The out-of-phase mode (dashed line) is not excited.

where $A$ is the cross-sectional area of the layers, assuming a uniform background magnetic field $B_{0}$ parallel to $E_{0}{ }^{2}$ Writing the power lost from the stack as $P_{\text {loss }}=$ $\omega U_{\text {osc }} / Q$, where

$$
U_{\mathrm{osc}}=\int d V \epsilon\left\langle|E|^{2}\right\rangle=\frac{N A}{\omega} \frac{1}{2}\left(\frac{1}{n_{1}}+\frac{1}{n_{2}}\right) E_{0}^{2}
$$

is the energy stored in this mode [27], we obtain a cycleaveraged power loss of

$$
\begin{aligned}
\left\langle P_{\mathrm{abs}}\right\rangle & =\frac{\left\langle P_{\text {int }}\right\rangle^{2}}{\left\langle P_{\text {loss }}\right\rangle} \simeq \frac{Q}{\omega} \frac{\left\langle P_{\text {int }}\right\rangle^{2}}{\left\langle U_{\text {osc }}\right\rangle} \\
& =\frac{1}{2}\left(g a_{0} B_{0}\right)^{2} Q A N\left(\frac{1}{n_{1}}+\frac{1}{n_{2}}\right)\left(\frac{1}{n_{2}}-\frac{1}{n_{1}}\right)^{2} \\
& =g^{2} B_{0}^{2} \frac{\rho_{\mathrm{DM}}}{m^{2}} Q A N\left(\frac{1}{n_{1}}+\frac{1}{n_{2}}\right)\left(\frac{1}{n_{2}}-\frac{1}{n_{1}}\right)^{2},
\end{aligned}
$$

where $\rho_{\mathrm{DM}} \simeq 0.3 \mathrm{GeV} / \mathrm{cm}^{3}$ is the local dark matter density and $m$ is the axion mass. This corresponds to solving for $E_{0}$ such that $\left\langle P_{\text {int }}\right\rangle=\left\langle P_{\text {loss }}\right\rangle$. The "quality factor" $Q$ depends on the stack's surroundings. For an "open cavity" setup corresponding to a stack of layers in air (i.e., none of the photons emitted by the layers are reflected back), the power loss is proportional to the area of the end-caps, so $Q \propto N$. However, the precise value depends on the form of the stack-air interfaces. For example, if the left-hand end of the stack were an $n_{1}$ layer, and the right-hand end an $n_{2}$ layer, as in Fig. 4, then $Q=2 N\left(1 / n_{1}+1 / n_{2}\right)$.

\footnotetext{
${ }^{2}$ There is an ambiguity coming from the integral outside the stack, which cancels over a period, but this is only a $\sim 1 / N$ fractional contribution [26].
} 


\section{Other configurations}

The "half-wave stack" configurations considered above have many useful special properties. For generic layer profiles, there are "bandgaps" around each $k=0$ point, as illustrated in Fig. 3. At frequencies within the bandgap, there are no periodic mode solutions-for a finite set of layers, incident modes at these frequencies are exponentially attenuated inside the material. Since the converted photons produced by the DM must be close to these bandgap edges, this can lead to complicated behavior (in particular, to very narrow mass range coverage). However, the bandgaps around the $k=0$ points for half-wave stacks vanish (right-hand panel of Fig. 3). A related advantage of this configuration is that such a stack has high transparency at frequencies close to zero Bloch momentum. Consequently, it is easy for photons produced by one stack to pass through another at a nearby frequency (Sec. II E). ${ }^{3}$

Another point is that, for a stack terminated on one end by a mirror (as per Fig. 1), the mirror's boundary condition means that there is only one free mode at a given frequency, which is a standing wave. The absorbed power will then be controlled by the $E$ integral of this mode. However, while this can be very small at the central frequency, if the mirror selects the "wrong" Bloch mode, we can go to a close-by frequency, $\delta \omega \sim \omega / N$, and recover an order- $N$ overlap. If the mirror selects out the correct Bloch mode, e.g., if the left-hand side of Fig. 4 were replaced by a mirror, then Eq. (8) applies. For a stack-air interface at an $n_{2}$ layer, $Q=4 N\left(1 / n_{1}+1 / n_{2}\right)$.

It is simple to modify $Q$ further by placing (partially) reflecting surfaces (e.g., other dielectric layers) at the ends of a stack; effectively, placing it inside a cavity. However, as Sec. II C shows, increased $Q$ is always compensated for by decreased mass range coverage (though this can still be helpful in terms of signal discrimination and signalto-noise).

\section{DM velocity distribution}

The DM in our galaxy is expected to have a virialized velocity distribution, with typical velocity $v \sim 10^{-3}$. Consequently, the DM field has a coherence length $l_{\text {coh }} \sim 1 /(v m)$. This means that photon emission from points further apart than $l_{\text {coh }}$ adds incoherently, when averaged over long times. Thus, stacks of $N \gtrsim v^{-1} \sim 10^{3}$ periods will no longer have peak conversion power $\sim N^{2}$. This can be verified by explicit computations, as in [36]. ${ }^{4}$ However, as we discuss in Sec. II C, the converted power

\footnotetext{
${ }^{3}$ These configurations, first considered in [35], are referred to by the MADMAX collaboration as "transparent mode" [26].

${ }^{4}$ Note that the expressions and plots shown in [36] are for emission from only one end of a (non-mirror-backed) stack; the deviation of these quantities from their zero-velocity values can be significantly greater than the deviation in the overall converted power.
}

averaged over DM masses is almost unaffected by the velocity distribution.

Another effect of the DM velocity distribution is on the angular distribution of converted photons. Since the transverse momentum of a converted photon is the same as that of the DM quantum, the emitted photons will be distributed within a cone of opening angle $\sim 10^{-3}$ around the $z$ axis. This is important in terms of focusing the emitted photons (Sec. III B), and could potentially be used post-discovery to determine the velocity distribution of the DM $[31,37,38]$. The DM velocity distribution also affects the frequency spectrum of converted photons, and (in a non-mirrorbacked stack) the backwards vs forwards emission rates [39]; if a signal were seen, these could be checked against each other for consistency with a DM signal origin.

\section{B. Dark photon conversion}

In addition to axions, light vector DM is another natural candidate for dielectric haloscope searches. A "dark photon" coupled to the SM through kinetic mixing with the photon has an unusually large window of open parameter space, since plasma effects suppress its emission from stars [40-42], and it does not mediate long-range forces between neutral matter. Also, unlike spin-0 DM candidates, vector $\mathrm{DM}$ has a polarization direction, and can convert to transverse photons even in a homogeneous target without velocity suppression. As discussed in Sec. V, a dark matter abundance of such vectors can naturally be produced in the early universe via inflation.

Suppose that dark matter consists of a dark photon $A^{\prime}$, with

$\mathcal{L} \supset-\frac{1}{4} F_{\mu \nu} F^{\mu \nu}-\frac{1}{4} F_{\mu \nu}^{\prime} F^{\prime \mu \nu}+\frac{1}{2} m^{2} A^{\prime 2}+J_{\mathrm{EM}}^{\mu}\left(A_{\mu}+\kappa A_{\mu}^{\prime}\right) ;$

this is equivalent, after field redefinition, to the usual "kinetic mixing" interaction $-\frac{1}{2} \kappa F_{\mu \nu} F^{\prime \mu \nu}$. Solving for the fields in a uniform dielectric, an oscillatory $A_{0}^{\prime}$ background field induces a corresponding standard model (visible) electric field $E_{\text {vis }}=-\kappa \frac{\chi}{\epsilon} E_{0}^{\prime}$, where $E_{0}^{\prime}=\partial_{t} A_{0}^{\prime}$ is the dark electric field, and $\chi=\epsilon-1$ is the polarizability [35]. This is in comparison to the axion-induced electric field $E_{\mathrm{vis}, \mathrm{a}}=$ $-g a B_{0} / \epsilon$ [26]. Since the boundary conditions at a dielectric interface are the same in both cases, the dark-photon induced power (per unit area, from a single interface) is given by

$\left(E_{\mathrm{vis}}^{1}-E_{\mathrm{vis}}^{2}\right)_{\|}=\left(\frac{\chi_{1}}{\epsilon_{1}}-\frac{\chi_{2}}{\epsilon_{2}}\right) \kappa\left(E_{0}^{\prime}\right)_{\|}=\left(\frac{1}{\epsilon_{2}}-\frac{1}{\epsilon_{1}}\right) \kappa\left(E_{0}^{\prime}\right)_{\|}$,

where $\|$ denotes the part parallel to the interface. Thus, the conversion rate for a dark photon amplitude $E_{0}^{\prime}$ is equivalent to that for an axion amplitude $a_{0}$ with $\kappa\left(E_{0}^{\prime}\right)_{\|}=$ $g a_{0} B_{0}$. For a half-wave stack, 
$\left\langle P_{\mathrm{abs}}\right\rangle=\kappa^{2} \sin ^{2} \theta \rho_{\mathrm{DM}} Q A N\left(\frac{1}{n_{1}}+\frac{1}{n_{2}}\right)\left(\frac{1}{n_{2}}-\frac{1}{n_{1}}\right)^{2}$,

where $\theta$ is the angle of $E_{0}^{\prime}$ from the layer normals. This angle will be approximately constant over the coherence timescale of the DM field, $t_{\mathrm{coh}} \sim m^{-1} v^{-2}$, and will vary over longer timescales; for an isotropic DM velocity distribution, the long-time average of $\sin ^{2} \theta$ is $2 / 3$. One difference between the dark photon and axion cases is that, for the latter, the polarization of the emitted photons is set by the $B_{0}$ field, while for a dark photon, it is set by the DM itself.

From Eq. (11), the dark photon to photon conversion rate is independent of $m$, for a given target volume (since the length of an $N$-period stack is $\propto \mathrm{m}^{-1}$ ). This may naively be worrying, since the dark photon has to decouple from the $\mathrm{SM}$ in the $m \rightarrow 0$ limit. For cavity experiments such as those proposed in [21], this manifests itself as a $(m L)^{2}$ suppression of the conversion rate, where $L$ is the scale of the experiment, for $m L \ll 1$. However, in our case, the width of the layers is set by $m^{-1}$, so when our expressions are valid, the experiment is automatically much larger than the DM Compton wavelength.

\section{Frequency-averaged power absorption}

Considering a spatially-uniform DM field oscillation to begin with, a natural expectation is that the fractional range in frequencies over which we get $\sim N^{2}$ converted power [Eqs. (8), (11)] is $\sim 1 / Q$. For example, a change $\delta \omega / \omega \sim$ $1 / N$ leads to a $\sim 1$ change in phase across $N$ layers, destroying the coherent addition. Figure 5 shows this scaling for example values of $N$. Thus, while the peak conversion power is $\sim N^{2}$, the averaged power over an $\mathcal{O}(1)$ range in frequencies is $\sim N$, and is not coherently enhanced. If we do not know the DM mass, which could a priori be anywhere in a wide interval, then the frequency-averaged power controls how fast we can scan over a range of masses (for a low-background experiment).

We can see the $\sim N$ scaling in more generality from an "impulse response" argument (for clarity, we take the example of axion DM here; the same arguments apply to a dark photon). For a target consisting of a set of thin interfaces between uniform layers, a sufficiently short "pulse" $a(t)$ of the DM field must, immediately after its arrival, result in only local field disturbances around the interfaces: influences have not had time to propagate further, and a uniform lossless dielectric does not absorb any energy. In the linear regime, the response of the target to a superposition of DM signals is the superposition of the responses to each signal; accordingly, we can decompose the pulse into sinusoidal components. Analyzing the response of a single interface $i$ to a sinusoidal DM oscillation, we find that the cycle-averaged power absorbed, per unit area, is

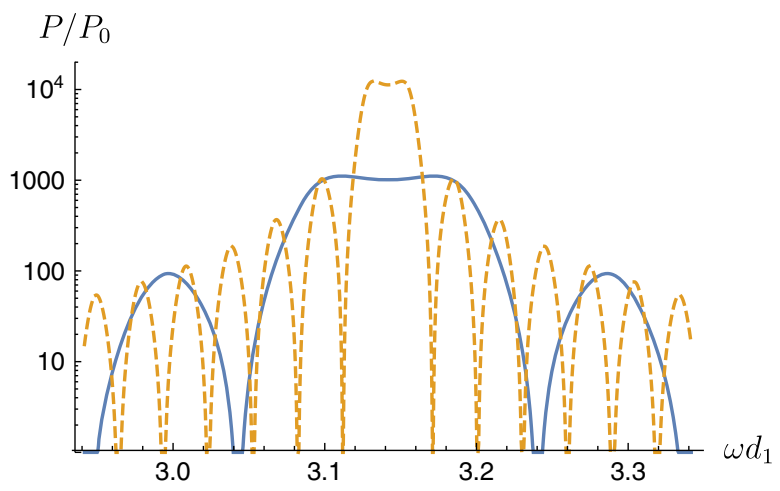

FIG. 5. Power absorbed from spatially-uniform DM oscillation, as a function of dark matter frequency $\omega$, for a half-wave stack of layers with refractive indices $n_{1}=1, n_{2}=2$, showing results for 30 (solid blue) and 100 (dashed orange) periods. As discussed in the text, the peak conversion power increases as $N^{2}$, where $N$ is the number of layers, but the mass range over which this holds decreases as $1 / N$. The reference power is defined by $P_{0} \equiv$ $g^{2} B_{0}^{2} \rho_{\mathrm{DM}} \omega^{-2}$, for axion $\mathrm{DM}$ in a uniform background magnetic field $B_{0}$.

$$
\frac{\left\langle P_{\mathrm{abs}}\right\rangle_{i}}{A}=\frac{\left(g a_{0} B_{0}\right)^{2}}{2}\left(\frac{1}{n_{1}}+\frac{1}{n_{2}}\right)\left(\frac{1}{n_{2}}-\frac{1}{n_{1}}\right)^{2},
$$

where $n_{1}$ and $n_{2}$ are the refractive indices on either side of the interface [26]. So, for a pulse $a(t)$ shorter than $\sim \min \left\{1 /\left(n_{i} d_{i}\right)\right\}$, for which the power absorbed should sum incoherently across different interfaces, the total energy absorbed (per unit area) is

$$
\begin{aligned}
\frac{U_{\mathrm{abs}}}{A} \simeq & \left(\int d \omega|\tilde{a}(\omega)|^{2}\right) \\
& \times \frac{g^{2} B_{0}^{2}}{2} \sum_{i}\left(\frac{1}{n_{1}}+\frac{1}{n_{2}}\right)\left(\frac{1}{n_{2}}-\frac{1}{n_{1}}\right)^{2},
\end{aligned}
$$

where $\tilde{a}(\omega)$ is the Fourier transform of the pulse $a(t)$, and the sum runs over interfaces $i$. If there is refractive index structure on significantly smaller scales, that can be treated as an effective medium of averaged refractive index. Since, in the linear regime, the power absorbed depends only on the power spectrum of the DM signal, and not on the relative phases of different frequency components, we can infer that for a general DM signal, with power spectral density $S_{a a}(\omega)$, the long-time average power absorbed, per unit area, is

$$
\begin{aligned}
\frac{\left\langle P_{\mathrm{abs}}\right\rangle}{A} \simeq & \left(\int d \omega S_{a a}(\omega)\right) \\
& \times \frac{g^{2} B_{0}^{2}}{2} \sum_{i}\left(\frac{1}{n_{1}}+\frac{1}{n_{2}}\right)\left(\frac{1}{n_{2}}-\frac{1}{n_{1}}\right)^{2},
\end{aligned}
$$

if $S_{a a}(\omega)$ is a broad distribution over the appropriate frequency range, and the refractive indices do not 
appreciably change over this frequency range. ${ }^{5}$ For periodic layer spacings, we can be more specific; in the case of a half-wave stack, the converted power is a periodic function of frequency (for given DM amplitude $a_{0}$ ) with period $2 \omega_{0}$, where $\omega_{0}$ is the half-wave frequency. Consequently, if we consider a flat power spectral density $S_{a a}(\omega)$, we find that the frequency-averaged conversion power is

$$
P_{\mathrm{av}}=\left(g a_{0} B_{0}\right)^{2} A N\left(\frac{1}{n_{1}}+\frac{1}{n_{2}}\right)\left(\frac{1}{n_{2}}-\frac{1}{n_{1}}\right)^{2},
$$

where we take the average to be over $0<\omega<2 \omega_{0}$. For a half-wave stack, most of this power is in the peak, at frequencies within $\sim 1 / Q$ of $\omega_{0}$, as illustrated in Fig. 5 .

If we do not know the DM mass, then since the DM is nonrelativistic, this corresponds to not knowing the oscillation frequency of $a$. Suppose that we have some number of different experimental configurations which, in combination, provide sensitivity over a $\mathcal{O}(1)$ range of $m$. Then, the total energy converted by all of the experiments, at the $m$ for which this is lowest, is at most the average energy converted over the whole range of $m$. This corresponds to taking $S_{a a}(\omega)$ approximately constant over the given frequency range, and summing across all of the experimental configurations. Thus, the minimum time-averaged powered converted by the set of configurations is at most

$$
\left\langle P_{\min }\right\rangle \lesssim \frac{\rho_{\mathrm{DM}}}{m_{\max }^{2}} g^{2} B_{0}^{2} \sum_{s} A_{s} \sum_{i}\left(\frac{1}{n_{1}}+\frac{1}{n_{2}}\right)\left(\frac{1}{n_{2}}-\frac{1}{n_{1}}\right)^{2},
$$

where $m_{\max }$ is the upper end of the mass range covered, and $s$ runs over the different stacks. For a dark photon, the corresponding expression has $g^{2} B_{0}^{2} / m_{\max }^{2}$ replaced by $\frac{2}{3} \kappa^{2}$. If the power converted as a function of mass is too "spiky," this bound maybe be difficult to attain; for example, Fig. 6 shows how random layer spacings result in sharp peaks at a fairly random set of frequencies, despite having similar frequency-averaged power (see Sec. II D). However, simple frequency profiles, such as that obtained from a half-wave stack, can easily be added together to obtain smooth coverage over an order-1 mass range, as illustrated in Fig. 9.

If an experiment is not background free, the frequencyaveraged conversion power will not be the only relevant quantity. Spending shorter times searching more, but narrower, frequency ranges will result in the same total number of signal photons produced over the whole lifetime of the experiment, but these all come within a shorter time, improving the signal to noise. However, as we will review

\footnotetext{
${ }^{5}$ This corresponds to the "area law" derived in [26], but extends it by giving an explicit expression for the frequencyaveraged power in terms of the refractive indices of the layers.
}

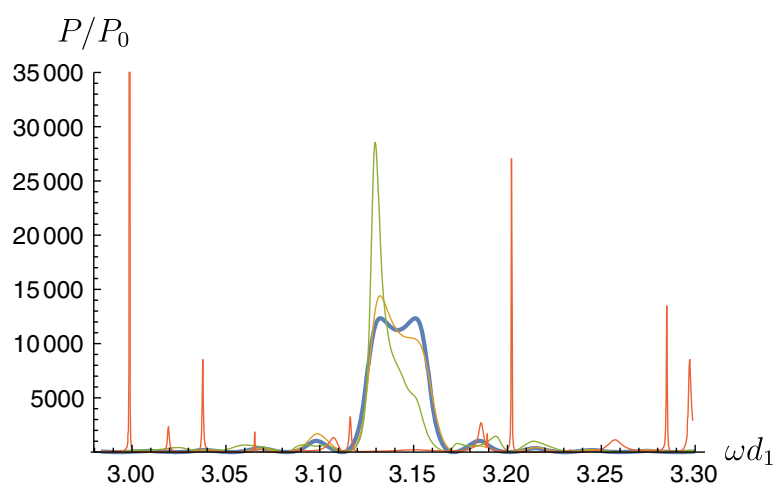

FIG. 6. Blue (thick) curve: power absorbed from spatially uniform DM oscillation at a single frequency $\omega$, for a 100-period half-wave stack with refractive indices $n_{1}=1, n_{2}=2$. The (thin) orange, green and red curves show the effect of introducing (uncorrelated) random thickness differences in the layers, with fractional deviation 0.01, 0.05, and 0.2. This illustrates that, when the fractional deviations are small compared to $\sim 1 / \sqrt{2 N}$, the effect on the frequency profile is small, whereas for larger deviations, the frequency profile changes completely, becoming spiky. The reference power is defined by $P_{0} \equiv g^{2} B_{0}^{2} \rho_{\mathrm{DM}} \omega^{-2}$, for axion DM in a uniform background magnetic field $B_{0}$.

in Sec. III, close to background-free photon detectors are possible over most of the frequency range we are considering. Consequently, in searching for a DM signal of unknown mass over a broad range, frequency-averaged power is a useful figure of merit. The simplest way to cover a large mass range is either to construct a set of stacks, each covering a small part of the range, or to construct a single stack with segments of different periodicities (a "chirped stack"). In the latter case, the transparency properties of the half-wave stack configuration discussed above are useful, as discussed in Sec. II E. ${ }^{6}$

The above results apply for spatially-uniform DM oscillations, i.e., $v_{\mathrm{DM}}=0$. As mentioned in Sec. II A 2, the DM velocity distribution, which is spread over a range $\delta v \sim 10^{-3}$, has a significant effect on the converted power when the layers are spaced over $\gtrsim 10^{3}$ wavelengths. However, by the same impulse response arguments as above, the frequency-averaged converted power is a local property for each interface. Since, for $\delta v \sim 10^{-3} \ll 1$, the scale over which the DM field varies is much larger than a signal photon wavelength, then if we average over a broad

\footnotetext{
${ }^{6}$ MADMAX [22,26] addresses the problem of achieving narrow mass coverage, $\delta \omega / \omega \ll 1 / N$, using a small number ( $N \sim$ tens) of slabs. This bandwidth is then scanned by physically repositioning the slabs. Their experiment is limited by thermal backgrounds, so narrow mass coverage improves the signal to noise. In our case, where such tuning may be difficult, but many more layers fit into a reasonable volume, the problem instead becomes covering a broad mass range without having to construct an enormous number of stacks; hence our emphasis on the halfwave stack.
} 
range of DM masses, the total power converted by all of the interfaces is still given by Eq. (15), to a good approximation. If we look at the total converted power as a function of DM mass, then the effect of the velocity distribution is to spread this out (by $\sim \delta v$ in fractional mass range), while almost preserving its mass-averaged value.

\section{Tolerances}

As discussed above, periodic layers have the advantage, compared to more random configurations, that their converted power is a smoother function of frequency (i.e., of DM mass), especially in the case of a half-wave stack. However, imperfections in the manufacturing process will result in some unintended variation in layer properties.

Considering flat, parallel interfaces, if the total accumulated phase error across all of the layers in a half-wave stack is $\gtrsim 1$, then we expect the frequency profile to change significantly (though, as per above, the frequencyintegrated power will stay approximately constant). Such deviations could arise from a combination of modified layer thicknesses and refractive indices. If deviations in different layers are uncorrelated, per-layer fractional deviations of up to $\sim 1 / \sqrt{2 N}$ will not significantly affect the profile (as is also derived in [26]). This is illustrated in Figure 6, which also shows an example of the highly modified "spiky" profile resulting from larger random deviations.

If we allow position-dependent thickness and/or index variations, resulting in nonplanar layers, then the same $\lesssim 1 / \sqrt{2 N}$ condition on uncorrelated fractional deviations is required at each position. This also ensures that the emitted photons are kept within the cone set by the DM velocity, with opening angle $\sim 10^{-3}$, as required for optimum focussing; while the layers may be "bumpy," the bumps have subwavelength height, and diffraction ensures that the overall emission is still collimated. As an additional point, we do not necessarily require that the fractional deviation condition applies strictly over the whole area of the stack. Emission from areas of the stack separated by more than a DM coherence length adds incoherently-therefore, to avoid a spiky frequency profile or uncollimated emission, we only need the fractional deviation condition to hold within these small areas. If layer thicknesses and/or indices change smoothly over larger distance scales, then different cross-sectional pieces of the stack effectively have different central frequencies, and add incoherently (as illustrated in the blue curve of Fig. 9).

The complicated frequency profiles of randomly-spaced layers occur because of their effect on photon propagation. In a random medium, instead of the definite bandgaps of a periodic medium, the frequency range corresponding to the inverse scale of variation becomes a "pseudogap," in which photons propagate diffusively $[43,44]$. The very long "effective path length" for a photon to escape the layers means that a very small change in frequency can significantly change the photon mode, leading to a very quickly-varying absorption rate with frequency.

So far, we have used the approximation of a lossless dielectric. However, a real material will absorb some of the light passing through it. Considering a photon mode in the layers, absorption will become important when the damping rate is $\gtrsim \omega / Q$, where $Q$ is the mode's quality factor. The half-wave stacks considered above have $Q \sim N$, so if the imaginary part of the refractive index is $\lesssim 1 / N$, then absorption will not be important. Configurations with narrower frequency peaks will be correspondingly more affected.

\section{E. Chirped stacks}

As we increase the number of layers in a half-wave stack, the frequency range covered decreases as $1 / N$. It is possible to increase the frequency range covered by a single stack, by having different parts of it correspond to half-wave stacks at different frequencies. If this variation is in a direction parallel to the layers, and is over a scale significantly larger than the DM coherence length, then the effect is equivalent to running multiple stacks side-by-side.

The variation can also be perpendicular to the layers, e.g., by gradually changing the layer spacings and/or refractive indices from layer to layer, as illustrated in Fig. 7. We refer to this configuration as a "chirped stack." It is important that, over the scale of a few layers, the stack is close to half-wave, since other configurations are not transparent to nearby frequencies, and result in very spiky frequency profiles. Figure 8 plots the transmittance of illustrative half-wave stacks, as a function of frequency, showing how they can be close to transparent over an order1 range of frequencies around their central frequency. In order that the emission from the layers at one end of a stack is mostly transmitted through the layers at the far end, the decrease in phase depths should be $\lesssim 30 \%$. The interference effects arising from imperfect transmittance result in a spiky frequency profile, as shown in Fig. 9, but for a wide range of parameters, the troughs are not large enough to be problematic.

The simplest ways of varying the layer spacings or indices, such as a smooth variation (as per Fig. 7), or

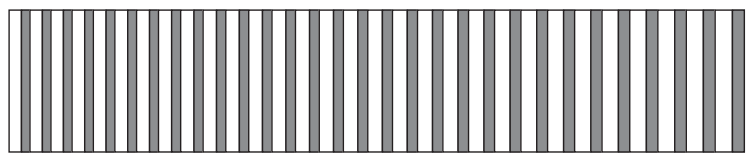

FIG. 7. Illustration of a "chirped stack" configuration, in which the layer spacings are gradually changed across the length of the stack. This example has 30 periods, with refractive indices $n_{1}=1, n_{2}=1.46$ (analogous to alternating gas/silica layerssee Sec. III A). The phase depths of adjacent layers are very close to equal, so that it locally approximates a half-wave stack, but the layer spacings at the right-hand end are 1.4 times the spacings at the left-hand end. 


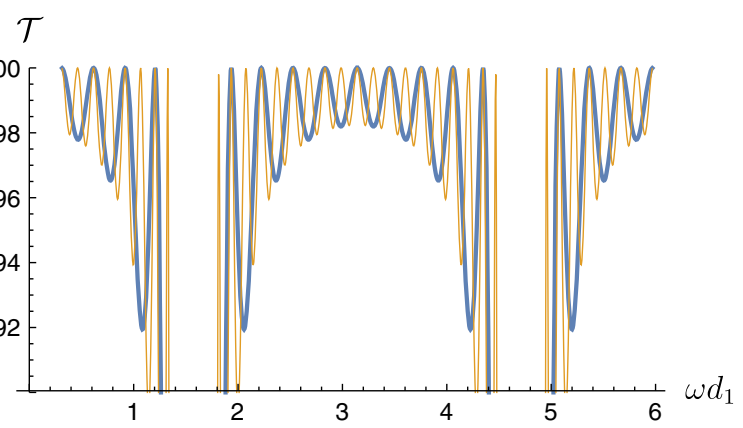

FIG. 8. Thick blue (thin orange) curve: transmittance of a 5-period (10-period) half-wave stack, with refractive indices $n_{1}=1, n_{2}=1.46$ (analogous to alternating gas/silica layerssee Sec. III A).

stacking different half-wave stacks on top of each other, all result in similar frequency profiles. It seems likely that this level of spikiness is inevitable, and cannot be ameliorated by clever choices of layer spacings. Another important point is that, unlike a half-wave stack, a chirped stack should not be placed on top of a mirror. Doing so results in very deep troughs in the frequency profile (effectively, from a part of the stack interfering with its reflection). If the mirror is placed far enough away from the stack that the light travel time is longer than the DM coherence time, then such cancellations can be avoided-however, this corresponds to $\gtrsim 10^{6}$ wavelengths, which would most likely be inconvenient on laboratory scales.

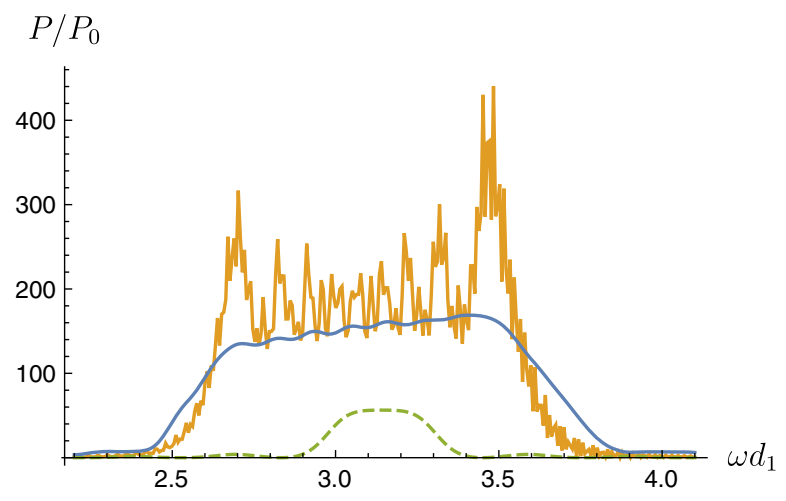

FIG. 9. Green dashed curve: power absorbed from spatially uniform DM oscillation at a single frequency $\omega$, by a half-wave stack of 10 periods with refractive indices $n_{1}=1, n_{2}=1.46$ (analogous to alternating gas/silica layers-see Sec. III A). Blue curve: incoherent sum of powers for 10 different 10-period halfwave stacks at different spacings, covering a fractional frequency range of $\sim 30 \%$. Orange (spiky) curve: power for a 100-period chirped stack, with layer spacings increasing by a factor 1.4 from one end to another (similar to Fig. 7). While this is spikier than the incoherent sum, it still results in an $\mathcal{O}(1)$ constant converted power across the same frequency range. The reference power is defined by $P_{0} \equiv g^{2} B_{0}^{2} \rho_{\mathrm{DM}} \omega^{-2}$, for axion DM in a uniform background magnetic field $B_{0}$.

\section{EXPERIMENTAL SETUP}

Figure 1 shows a sketch of the experimental setup outlined above; a stack of dielectric layers is placed in a shielded volume, and then photons emitted normal to the layers are focused onto a small detector. A dielectric stack in free space will emit equally in both directions; to facilitate detection, one end is terminated with a mirror. For dark photon DM, this setup is sufficient, while for an axion-photon coupling, a large background $B$ field parallel to the layers would be introduced. In this section, we develop this outline into a more detailed illustration of how such an experiment might be implemented.

To recap the most important properties of such a setup, the emitted photon power for $N$ periods of alternating dielectrics, with refractive indices $n_{1}, n_{2}$, at the frequency $m_{0}$ for which they form a half-wave stack (see Fig. 4),

$$
m_{0}=\frac{\pi}{n_{1} d_{1}}=\frac{\pi}{n_{2} d_{2}}
$$

is

$$
\begin{aligned}
\left\langle P_{\mathrm{abs}}\right\rangle & \simeq g^{2} B_{0}^{2} \frac{\rho_{\mathrm{DM}}}{m^{2}} Q A N\left(\frac{1}{n_{1}}+\frac{1}{n_{2}}\right)\left(\frac{1}{n_{2}}-\frac{1}{n_{1}}\right)^{2} \\
& =g^{2} B_{0}^{2} \frac{\rho_{\mathrm{DM}}}{m} \frac{Q V}{\pi}\left(\frac{1}{n_{2}}-\frac{1}{n_{1}}\right)^{2},
\end{aligned}
$$

where $Q$ is the quality factor of the mode in the layers, and $V$ is the volume of the stack. This expression is valid as long as the depth of the stack is smaller than a DM coherence length, corresponding to $N \lesssim v^{-1} \sim 10^{3}$ (See Sec. II A 1). The quality factor depends on how the layers are terminated, but is $\propto N$. For example, the setup drawn in Fig. 1, with a mirror on one side and air on the other, has $Q \simeq 4 N\left(1 / n_{1}+1 / n_{2}\right)$. For a dark photon, the expression is

$$
\left\langle P_{\text {abs }}\right\rangle=\frac{2}{3} \kappa^{2} m \rho_{\mathrm{DM}} \frac{Q V}{\pi}\left(\frac{1}{n_{2}}-\frac{1}{n_{1}}\right)^{2},
$$

after averaging over many DM coherence times. These expressions apply over a fractional frequency range $\sim 1 / Q$; the converted power as a function of frequency is nonLorentzian (see Fig. 5), and has peak power at frequencies $\sim 1 / Q$ away from the center frequency $m_{0}$.

The mass-averaged conversion power from a half-wave stack, over the DM mass range $0<m<2 m_{0}$, is

$$
P_{\mathrm{av}} \simeq\left\{\begin{array}{ll}
2 g^{2} B_{0}^{2} \frac{\rho_{\mathrm{DM}}}{m_{0}} \frac{V}{\pi}\left(\frac{1}{n_{2}}-\frac{1}{n_{1}}\right)^{2} & (\text { axion }) \\
\frac{4}{3} \kappa^{2} m_{0} \rho_{\mathrm{DM}} \frac{V}{\pi}\left(\frac{1}{n_{2}}-\frac{1}{n_{1}}\right)^{2} & (\mathrm{DP})
\end{array},\right.
$$

where DP stands for dark photon. If we construct $N_{s}$ different half-wave stacks, spaced to cover a frequency range $\Delta \omega$ with central frequency $m_{0}$, then the converted power averaged over the $\Delta \omega$ frequency range is 


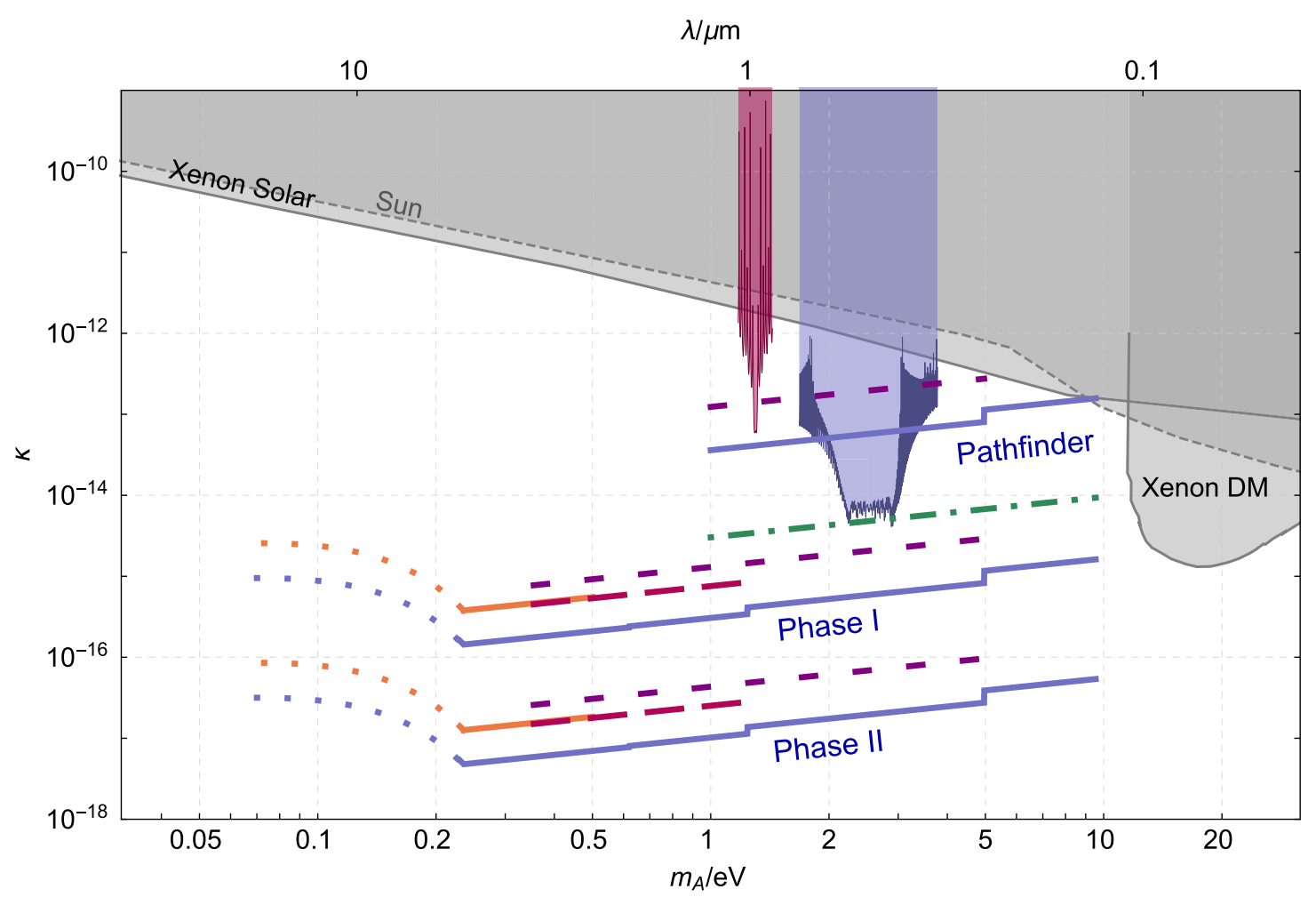

FIG. 10. Sensitivity to dark photon dark matter, in terms of the kinetic mixing parameter $\kappa$. The different experimental configurations are described in Table I. The reach is shown for a combination of half-wave stacks at different spacings, enabling smooth coverage of the mass range (Sec. III), and is a factor $\sim 2$ deeper than the peak reach for a single half-wave stack. We assume an integration time of $10^{6} \mathrm{~s}$ for each stack. We also show the sensitivity curves for two example stacks, consisting of alternating air $/ \mathrm{Si}_{3} \mathrm{~N}_{4}$ layers: a $N=30$ halfwave stack (red) with Pathfinder parameters (in particular, a detector DCR of $10^{-3} \mathrm{~Hz}$ ), and a wider-band $N=100$ "chirped" stack (blue), as per Figure 9, with Phase I parameters. The different colors/styles of curves correspond to different alternating dielectric pairs: $\mathrm{Ge} / \mathrm{NaCl}$ (solid, orange), $\mathrm{SiO}_{2} / \mathrm{GaAs}$ (long-dashed, red), $\mathrm{Si}_{3} \mathrm{~N}_{4} / \mathrm{SiO}_{2}$ (short-dashed, purple), doped $\mathrm{SiO}_{2}$ (dot-dashed, green). The solid blue lines correspond to alternating air/dielectric structures, for (from low to high frequencies) $\mathrm{Ge}_{2} \mathrm{Si}, \mathrm{Si}_{3} \mathrm{~N}_{4}, \mathrm{SiO}_{2}$. Different assumptions about the DM velocity distribution will only have a small effect on sensitivity (see Sec. III E). The dotted reach at low energy is an estimate of single-photon detector sensitivity in the IR (Sec. III B). Gray regions indicate current constraints from direct detection experiments $[45,46]$ and astrophysical measurements $[47,48]$.

$P_{\text {sum }} \simeq N_{s} \frac{2 m_{0}}{\Delta \omega} P_{\text {av }}$ (if all of the stacks have the same number of layers $N$, and $\Delta \omega \ll \omega)$. Since $N_{s} \simeq Q \Delta \omega / m_{0}$ stacks are required to obtain a smooth frequency profile, $P_{\text {sum }} \simeq$ $2 Q P_{\mathrm{av}}$. For mirror-backed stacks, with $Q \simeq 4 N\left(1 / n_{1}+\right.$ $\left.1 / n_{2}\right)$, this gives the signal power in our experiment,

$$
P_{\text {sum }} \simeq\left\{\begin{array}{ll}
8 g^{2} B_{0}^{2} \frac{\rho_{\mathrm{DM}}}{m_{0}^{2}} A N^{2} \frac{\left(n_{1}^{2}-n_{2}^{2}\right)^{2}}{n_{1}^{4} n_{2}^{4}} & \text { (axion) } \\
\frac{16}{3} \kappa^{2} \rho_{\mathrm{DM}} A N^{2} \frac{\left(n_{1}^{2}-n_{2}^{2}\right)^{2}}{n_{1}^{4} n_{2}^{4}} & (\mathrm{DP})
\end{array} .\right.
$$

These expressions can be used to estimate the sensitivity for a set of half-wave stacks covering some DM mass range, Figs. 10 and 11.

In the following, we consider three illustrative stages for the experiment (see Table I). The first, a "pathfinder," would be the simplest that could still probe new parameter space; it would aim to detect dark photon DM, could be run at room temperature, and would use readily-available detectors and layer fabrication methods. Phase I would aim to explore significant new dark photon parameter space, and start gaining sensitivity to new axion parameter space. This would require cooling the target to cryogenic temperatures, using cryogenic detectors, and using $\mathcal{O}(100)$ high-contrast layers, as well as operating with a large background $B$ field for the axion search. Phase II would aim to cover significant new axion and further dark photon parameter space, using a larger volume of layered material.

\section{A. Dielectric materials}

As discussed above, the proposed experiment requires periodic structures with a significant refractive index contrast; for lower frequencies, these need to operate at cryogenic temperatures. In this section, we summarize the requirements on material properties necessary for our setup.

The layers should be transparent at the relevant frequencies to avoid losses; transparency windows, typically extending up to the bandgap energy, are listed in table II. For example, transmittance in excess of $99.9 \%$ 


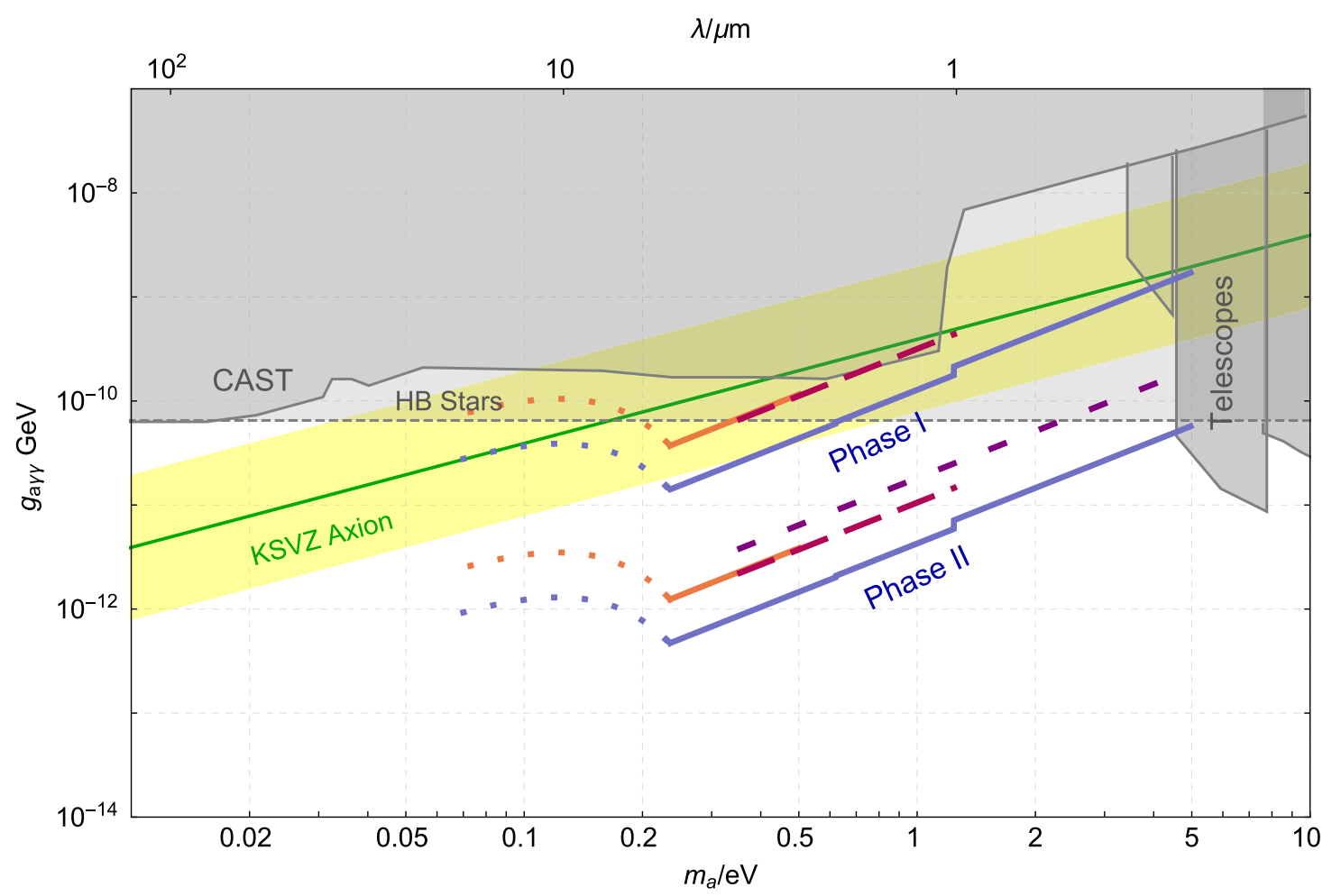

FIG. 11. Sensitivity to axion dark matter, in terms of its coupling to photons $g_{\text {ary }}$. The different experimental configurations are described in Table I. The reach is shown for a series of dielectric stacks at different spacings, enabling smooth coverage of the mass range (Sec. III). We assume an integration time of $10^{6} \mathrm{~s}$ for each stack. The different colors/styles of curves correspond to different alternating dielectric pairs: $\mathrm{Ge} / \mathrm{NaCl}$ (solid, orange), $\mathrm{SiO}_{2} / \mathrm{GaAs}$ (long-dashed, red), $\mathrm{Si}_{3} \mathrm{~N}_{4} / \mathrm{SiO}_{2}$ (short-dashed, purple), doped $\mathrm{SiO}_{2}$ (dot-dashed, green). The solid blue lines correspond to alternating air/dielectric structures, for (from low to high frequencies) $\mathrm{Ge}_{\mathrm{Si}} \mathrm{Si}, \mathrm{Si}_{3} \mathrm{~N}_{4}$. The dotted reach at low energy is an estimate of single-photon detector sensitivity in the IR (Section III B). Gray regions indicate current constraints from CAST [49], stellar cooling [48,50,51], and axion to photon decays [52]. The KSVZ axion line is shown as a guideline; SN 1987A constraints on the nuclear coupling of the QCD axion limit the mass to be $m_{a} \lesssim 60 \mathrm{meV}$ [53,54], assuming that its derivative couplings to nucleons are not suppressed.

at wavelengths above $250 \mathrm{~nm}$ is demonstrated for silica with mm thickness [55].

An interesting phenomenological property of crystal dielectrics is that the refractive index decreases with increasing bandgap energy $\omega_{0}: n^{4} \omega_{0} \sim 100 \mathrm{eV}[65,66]$. Thus with common dielectrics it is easier to achieve high sensitivity at lower frequencies.

Another requirement comes from the fact that, in order to suppress thermal backgrounds at frequencies $\lesssim \mathrm{eV}$, it will be necessary to cool the dielectric stack to cryogenic temperatures (see Sec. III D 1). For the layers to be stable under this temperature change, the materials should ideally have similar thermal expansion properties. One possibility would be to use the same host material-for example, the widely used silica $\left(\mathrm{SiO}_{2}\right)$-with index-raising [e.g., Germania $\left(\mathrm{GeO}_{2}\right)$ ] and index-lowering [e.g., Boron trioxide $\left(\mathrm{B}_{2} \mathrm{O}_{3}\right)$ and Fluorine $\left.(\mathrm{F})\right]$ dopants for the alternating layers [67-69]. A refractive index contrast of up to $10 \%$ can be achieved without significantly altering the mechanical and thermal properties of the material [70,71]. This procedure has been studied extensively e.g., to improve the performance of optical fibers at low temperatures [72], which rely on a significant core-vs-cladding refractive index contrast created by adding dopants to the host material in the fiber core $[67,73,74]$.

For an improved version of the experiment, materials with $\mathcal{O}(1)$ different refractive indices should be employed: maximum dark matter to photon conversion is achieved when the dielectric materials have indices of refraction $n_{1}=1, n_{2} \gg 1$ [Eq. (20)]. Thus, the highest power results when alternating air or vacuum with materials with high refractive index such as silicon $(\mathrm{Si}, n \sim 3.4)$. Layering alternating dielectrics may be more mechanically robust, in which case pairs such as silica $\left(\mathrm{SiO}_{2}, n \sim 1.46\right)$ and gallium arsenide (GaAs, $n \sim 3.8$ ), commonly used in the semiconductor industry [71], can achieve $20 \%$ of maximum power. It will be necessary to demonstrate that at least tens of alternating layers can be constructed and withstand the thermal stresses of cooling to cryogenic temperatures $[63,75]$.

The relevant technologies to create layered structures vary depending on the scale of the desired spatial periodicity, set by the dark matter mass. For DM in the $0.1-10 \mathrm{eV}$ mass range, corresponding to layer thicknesses $\sim 0.1-10 \mu \mathrm{m}$, possible 
TABLE I. Summary of nominal experimental parameters for the different phases of the experiment. As discussed in Sec. II D, the fractional variation in layer thicknesses should be $\lesssim 1 /(\sqrt{2 N})$ for an $N$-period stack. The temperature of the layers for the pathfinder phase of the experiment can be either $200 \mathrm{~K}$, which matches the operational temperature of the PIXIS CCD photon detector (Sec. III B), or as high as room temperature. The number of (mirror-backed) half-wave stacks needed to provide smooth coverage over an $e$-fold in DM mass range is shown. Fewer stacks with broader frequency coverage could be used, at the cost of lower sensitivity; similarly, multiple stacks could be run simultaneously to reduce total integration time.

\begin{tabular}{lccc}
\hline \hline & Pathfinder & Phase I & Phase II \\
\cline { 2 - 3 } Signal & Dark photon & Dark photon \& axion & Dark photon \& axion \\
\hline Range $\left(m_{\mathrm{DM}} \& \lambda_{\text {Compton }}\right)$ & $(1 \mathrm{eV}, 10 \mathrm{eV})$ & $(50 \mathrm{meV}, 10 \mathrm{eV})$ & $(50 \mathrm{meV}, 10 \mathrm{eV})$ \\
& $(0.1 \mu \mathrm{m}, 1 \mu \mathrm{m})$ & $(0.1 \mu \mathrm{m}, 20 \mu \mathrm{m})$ & $(0.1 \mu \mathrm{m}, 20 \mu \mathrm{m})$ \\
Area $(A)$ & $(10 \mathrm{~cm})^{2}$ & $(10 \mathrm{~cm})^{2}$ & $(30 \mathrm{~cm})^{2}$ \\
Number of periods $(N)$ & 30 & 100 & 1000 \\
Temperature $\left(T_{\text {layer }}\right)$ & $200 \mathrm{~K}(300 \mathrm{~K})$ & $4 \mathrm{~K}$ & $4 \mathrm{~K}$ \\
Thickness $(d \sim N \lambda)$ & $(\sim 3 \mu \mathrm{m}, \sim 30 \mu \mathrm{m}$ & $(\sim 10 \mu \mathrm{m}, \sim 2 \mathrm{~mm})$ & $(\sim 100 \mu \mathrm{m}, \sim 20 \mathrm{~mm})$ \\
Stacks per $e$-fold & 150 & 400 & 4000 \\
Detector Dark Count $\left(\Gamma_{\text {DCR }}\right)$ & $\mathrm{mHz}(\mathrm{e} . \mathrm{g} ., \mathrm{CCD})$ & $10^{-5} \mathrm{~Hz}(\mathrm{e} . \mathrm{g} ., \mathrm{TES})$ & $10^{-5} \mathrm{~Hz}(\mathrm{e} . \mathrm{g} ., \mathrm{TES})$ \\
Detector Efficiency $(\eta)$ & 0.1 & 0.9 & 0.9 \\
Temperature $\left(T_{\text {detector }}\right)$ & $200 \mathrm{~K}$ & $100 \mathrm{mK}$ & $100 \mathrm{mK}$ \\
Magnetic Field (Axion) & N/A & $10 \mathrm{~T}$ & $10 \mathrm{~T}$ \\
\hline \hline
\end{tabular}

production processes include chemical vapor deposition (CVD), physical vapor deposition (PVD), spin coating, epitaxy, and sputtering (for a review, see [76]). These methods are well-established for such length scales and are employed in producing optics such as mirror and lens coatings, and semiconductor diode lasers [surface-emitting laser and the vertical-cavity surface-emitting laser (VCSEL)] [77-79], with tens of layers in the optical wavelength range

TABLE II. A list of materials that can be used to construct the layers for different dark photon and axion masses and their main optical and thermal properties [56-63]. The refractive index $(n)$ is the value at vacuum-wavelength $\sim 1.5 \mu \mathrm{m}$, at room temperature. The pathfinder phase of our experiment will be operated at 200-300 K, while Phase I and Phase II of our experiment will be operated at liquid helium temperatures. Refractive indices at those temperatures will be needed for manufacturing purposes. Coefficients of thermal expansion (CTE) at room temperature are also listed. A few of the elements have radioactive isotopes that can cause additional backgrounds in our experiment, and therefore require additional purification procedures.

\begin{tabular}{llllcl}
\hline \hline & & $\begin{array}{c}\text { Wavelength } \\
\text { range }(\mu \mathrm{m})\end{array}$ & $\begin{array}{c}\text { DM mass } \\
\text { range }(\mathrm{eV})\end{array}$ & $\begin{array}{c}\mathrm{CTE} \\
\left(10^{-6} / \mathrm{K}\right)\end{array}$ & Radioactive \\
\hline $\mathrm{SiO}_{2}$ & 1.46 & $(0.13,3.5)$ & $(0.35,9.5)$ & 0.55 & \\
$\mathrm{GaAs}$ & 3.8 & $(1,15)$ & $(0.08,1.2)$ & 5.7 & \\
$\mathrm{Ge}$ & 4.0 & $(2,17)$ & $(0.07,0.62)$ & 6.1 & \\
$\mathrm{NaCl}$ & 1.49 & $(0.2,20)$ & $(0.06,6.2)$ & 44 & ${ }^{36} \mathrm{Cl}[54,64]$ \\
$\mathrm{Si}_{3} \mathrm{~N}_{4}$ & 2.00 & $(0.25,8)$ & $(0.15,5)$ & 3.3 & \\
$\mathrm{Si}$ & 3.42 & $(1.1,9)$ & $(0.12,1.0)$ & 2.55 & \\
$\mathrm{MgF}_{2}$ & 1.41 & $(0.12,9.5)$ & $(0.13,10)$ & 13.7 & \\
$\mathrm{CaF}_{2}$ & 1.43 & $(0.15,9)$ & $(0.14,8.3)$ & 18.85 & \\
$\mathrm{ZnSe}$ & 2.4 & $(0.55,20)$ & $(0.06,2.3)$ & 7.1 & ${ }^{79} \mathrm{Se}[54]$ \\
$\mathrm{GaN}^{2}$ & 2.3 & $(0.37,13.6)$ & $(0.09,3.4)$ & 3.17 & \\
\hline \hline
\end{tabular}

commercially available. Acid-etching can potentially be used to achieve alternating dielectric/air structures $[80,81]$.

As discussed in Sec. II D, the structure should be close to flat and periodic. Each stack, once built, can be tested with an broadband laser beam, for example a Ti:Sapphire laser [82]. By measuring the transmitted and reflected wave from the stack, the resonant frequency as well as the normal direction of the layers can be measured to very high precision $[27,83,84]$.

Our calculations throughout have neglected dispersion, by assuming that the dielectric materials have a constant refractive index as a function of frequency. For the fairly small fractional frequency ranges covered by each stack, this is almost always a good approximation, since refractive index changes over $e$-fold frequency ranges within the bandgap are $\lesssim 5 \%$.

\section{B. Photon detection}

As discussed in Sec. IIIE, the existing constraints on axion or dark photon couplings mean that, even if they make up all of the dark matter, the photon conversion rate from a reasonably-sized target will be small. Accordingly, we will require a sensitive photon detector with a sufficiently low energy threshold, high photon efficiency, and low noise.

From Sec. II A, the DM momentum spread means that converted photons are emitted in a narrow cone (opening angle $\sim 10^{-3}$ ) around the normal to the layers. Consequently, the photons from a stack of cross-sectional area $A$ can be optically focused down to an area $\sim 10^{-6} A$ (for stack radii $\gtrsim \mathrm{cm}$, this is larger than a square wavelength, for the DM mass range we are considering). For a 
stack of area $(10 \mathrm{~cm})^{2}$, the detector must have area $\gtrsim(100 \mu \mathrm{m})^{2}$ to intercept $\mathcal{O}(1)$ of the signal photons. The required area could be decreased by various techniques, such as using a high-refractive-index concentrator on top of the detector, or having the signal photons bounce multiple times within a cavity.

The most commonly used photon detectors in our frequency range are charge-coupled devices (CCDs), which are found in a wide range of astronomical and laboratory applications. A close analogue to our low-signal-flux, longintegration-time setting is the ALPS "light shining through wall" experiment [85], which looks for very rare photonaxion-photon conversion events at optical frequencies. The PIXES CCD camera [86], used in the ALPS experiment and planned as a backup for the ALPS-II upgrade, operates at $\gtrsim-70^{\circ} \mathrm{C}$ and has $1024 \times 1024$ pixels with per-pixel area of $(13 \mu \mathrm{m})^{2}$, detection efficiency of $\eta \sim 10 \%$, and dark count rate $\Gamma_{\mathrm{DCR}} \sim \mathrm{mHz}$ for wavelengths shorter than $\sim \mu \mathrm{m}$. Accordingly, we adopt similar parameters for the pathfinder stage.

Detectors with similar frequency coverage $\left(m_{\mathrm{DM}} \gtrsim\right.$ $1.1 \mathrm{eV})$, better efficiency ( $\gtrsim 20 \%)$ and lower dark count rate in cryogenic environments $(0.1 \mathrm{mHz}$ per pixel) [87], are being developed for dark matter direct detection experiments based on liquid xenon. The per-pixel area of this detector is $50 \mu \mathrm{m} \times 50 \mu \mathrm{m}$, and arrays of $60 \times 60$ pixels have been demonstrated [88]. With optimization, these detectors can be ideal for transitioning between the pathfinder and phase I of our experiment.

To reach our phase I and phase II sensitivities, dark count rates of $\lesssim 10^{-5} \mathrm{~Hz}$ are required. These rates have been demonstrated for multiple detector technologies, including Transition Edge Sensors (TES) [89-94] (for a review, see [95]), Microwave Kinetic Inductance Detectors (MKIDs) [96-98], and nanowires [99]. The efficiency of these detectors can be quite high: for example, a TES can be coupled to photon modes using specially designed coatings in a narrow range of frequencies, reaching efficiencies of $\eta \gtrsim 95 \%$, with demonstrated $98 \%-99 \%$ detection efficiency for wavelengths between 0.6 and $2 \mu \mathrm{m}$ [91,92].

Achieving such low dark count rates generally requires small detectors. For example, a TES has an exponentially suppressed dark count rate above its energy resolution, but this energy resolution increases with the size of the TES; thus, to keep the dark count rate low, it is crucial that we are able to focus the signal to a small area, of order tens of microns on a side $[90,92,95]$. One possibility to achieve a larger total detector area is to multiplex multiple TES pixels; arrays of more than 200 pixels have been demonstrated [95].

For most of the energy range we cover, we assume that the DCR and other backgrounds (see Sec. III D) can be controlled to below $10^{-3} \mathrm{~Hz}$ for the pathfinder phase, and below $10^{-5} \mathrm{~Hz}$ for phases I and II. At DM masses below $\sim 0.2 \mathrm{eV}$, the energy of a signal photon is close to the currently achievable detector energy resolution. For the low-frequency regions of Figs. 10 and 11 (shown in dotted lines), we assume a TES-type noise curve with $\Gamma_{\mathrm{DCR}} \propto$ $0.1 \mathrm{~Hz} e^{-\omega^{2} / 2 \Delta E^{2}}$, with $\Delta E \sim 50 \mathrm{meV}$. This normalization gives a reach that approximately matches to the reach of a bolometer with noise equivalent power $\sim 10^{-20} \mathrm{~W} / \sqrt{\mathrm{Hz}}$ (demonstrated at lower energies $[95,100,101]$ ) at around $50 \mathrm{meV}$. We are not aware of whether such sensitivities have been demonstrated in the near- to mid-IR regime we are considering.

The signal power calculations above have used the framework of a classical DM field driving a classical signal photon mode. For the light bosonic DM production mechanisms discussed in Sec. V, the early-universe DM abundance is expected to take the form of a large occupation number coherent state, corresponding to a classicallike oscillation of the field, with a definite oscillation phase. Since then, the evolution of the coherent state has most likely maintained this coherence. Even though the occupation number of DM modes around Earth is less or comparable to 1 for $m \lesssim 20 \mathrm{eV}$, these will still be smallamplitude coherent states (like, e.g., an attenuated laser mode). The DM then excites the signal mode into a smallamplitude coherent state, which has mean occupation number given the classical power calculation, and Poissonian number statistics. Over timescales longer than the DM coherence time, variation of the DM field amplitude can lead to super-Poissonian photon detection fluctuations. If the DM is significantly spatially clumped, then these fluctuations can be very large; however, the most common assumption is that most of the galactic DM is smoothly distributed, in which case such fluctuations will average out over many coherence times.

If - due to some unknown mechanism-the DM field around Earth were in a very different quantum state, then we would still expect almost the same Poissonian statistics of detected signal photons. This is true whenever the probability of converting a DM mode to a photon is small. Hence, our sensitivity calculations should apply very generally. These considerations justify treating the DM as a classical-like background oscillation, as done in many light bosonic DM detection methods. Potential experimental differences between different DM quantum states can arise if e.g., we employ a phase sensitive amplification method, rather than pure photon counting.

\section{Scanning}

The simplest scanning mechanism would be if it were possible to change the refractive indices of the layers by an external perturbation. If the refractive indices could be changed by $\mathcal{O}(1)$, this would enable a single stack to cover an entire decade of frequency range. However, the optical materials of the types we have been considering typically have very small refractive index changes in response to reasonable external perturbations. For example, the rate of 
change of refractive index with temperature for silica, at $\sim \mu \mathrm{m}$ wavelengths, is $\lesssim 10^{-5} \mathrm{~K}^{-1}$ [60]. Especially at the lower end of our frequency range, where the layers' temperature needs to be low to suppress blackbody radiation, this does not allow significant scanning. Similarly, the refractive index changes due to applied electric fields (Kerr/Pockels effect), magnetic fields (Verdet effect) or strain (photoelastic/piezooptic effect) are generally too small for our purposes.

While birefringent materials can have different refractive indices for different propagation directions, there are only two distinct polarizations corresponding to a given propagation direction. Since the direction of photon emission is set (to within $\sim 10^{-3}$ ) by the layered structure of the material, changes that do not affect the layered structure itself (e.g., rotating the material relative to the $B$ field) will not result in significant scanning.

The opposite question, of whether we can tunably narrow the bandwidth of an existing stack, is also of interest. When backgrounds are important, narrowing the bandwidth improves the signal to noise, and it would also allow us to home in on a tentative signal. The simplest way to reduce the bandwidth is to increase the quality factor by placing e.g., a half-wave stack inside a "cavity." For example, if we place a quarter-wave stack of $M$ periods above a half-wave stack, this increases the $Q$ factor by $G=C^{M}$, up to manufacture and alignment accuracy (where $C$ depends on the dielectric contrast of the quarter-wave stack). By changing the separation between the quarter-wave and half-wave stacks over a distance $\sim N / G$ wavelengths, we can scan this narrowed bandwidth across the entire original bandwidth of the half-wave stack. This procedure would demand improved tolerances so as not to smear out the narrowed peaks - in particular, the separation between the plates should be the same across the whole area, to within $\sim N / G^{2}$ of a wavelength-and accurate positioning of the quarter-wave stack to within $\sim N / G^{2}$ wavelengths.

\section{Environmental backgrounds}

Since the flux of signal photons in our experiments would be very weak, it is important to be able to discriminate these from backgrounds. In this section, we will discuss the backgrounds from radioactivity, cosmic rays, and blackbody radiation, and the requirements these impose on our experimental design.

\section{Blackbody}

If the detector's field of view is at temperature $T$, the rate at which thermal photons within a small energy range $\Delta \omega$ of $\omega$ hit the detector is

$$
\Gamma_{\mathrm{BB}} \sim \frac{\Delta \omega \omega^{2}}{4 \pi^{2}} A_{\mathrm{det}} e^{-\omega / T},
$$

for $\omega \gg T$, where $A_{\text {det }}$ is the area of the detector. For the pathfinder, a room-temperature field of view $(T \sim 300 \mathrm{~K})$ gives small $(\lesssim \mathrm{mHz})$ dark-count rate for $\omega \gtrsim \mathrm{eV}$. Since $\omega \gtrsim 40 T$, we are well into the blackbody tail, and the minimum frequency giving the desired dark count rate depends only logarithmically on detector size. For phases I and II, we are interested in photon energies down to $\sim 50 \mathrm{meV} \simeq 600 \mathrm{~K}$, and similarly require $T \lesssim \omega / 40 \sim$ $15 \mathrm{~K}$. Since we are focusing the signal photons from the layers onto the detector, we require that at least the dielectric layers, and the surrounding shielding, are cooled to these temperatures. It would likely be practical to cool the target volume to $4 \mathrm{~K}$ using liquid helium.

The photodetectors we require for phases I and II are generally cryogenic, and need to be operated at temperatures $\ll 15 \mathrm{~K}$; for example, low-noise TESs are operated at $\sim 100 \mathrm{mK}$. To help achieve this, a cold filter could be placed between the layers and the detector, which is transparent to photons at the signal frequency, but blocks the lower-frequency thermal radiation.

\section{Cosmic rays and radioactivity}

In addition to blackbody photons, there will also be less frequent but more energetic background events. One source of these is cosmic rays. The sea-level cosmic ray flux is dominated by muons, with a rate of $\sim 1 /(10 \mathrm{~cm})^{2} / \mathrm{sec}$. These deposit $\sim 100 \mathrm{keV} / \mathrm{mm}$ as they travel through typical materials [102]. Radioactive decays in or near the experiment constitute another background. Laboratory materials will contain some preexisting (or cosmogenic) level of radioactive isotopes; when these decay, they can produce particle showers in the experiment.

The fact that all of the signal photons are focused onto a small detector, compared to a more uniform flux of shower particles (from decays or cosmics), improves our signal to background ratio. In addition, the fact that showers generally consist of many particles, while signal photons arrive one at a time, provides a discrimination strategy. Multiple detectors, either in the form of a pixel array or separate detectors, will generally register many simultaneous counts for a shower event, allowing that time interval to be vetoed. As long as $\mathcal{O}(1)$ of the observation time is not vetoed, these detectors can have higher dark count rates than the one employed for the signal photons.

If such veto schemes are not sufficient, then it may be possible to reduce the background rates directly. For cosmic rays, this could be accomplished by running the experiment deep underground. For radioactive decays, it may be possible to fabricate the experimental setup with radiologically pure materials. Taking the example of chlorine, the radioactive isotope ${ }^{36} \mathrm{Cl}$ has natural fractional abundance $7 \times 10^{-13}$, and half life $3 \times 10^{5}$ yrs [103]. This gives rise to an event rate of $\sim 10^{-3} \mathrm{~Hz}$ from a gram of chlorine. A much lower ${ }^{36} \mathrm{Cl} / \mathrm{Cl}$ ratio of $\sim 10^{-15}$ can be found in old ground water [64], which would reduce the background event rate 
below $\sim 10^{-5} \mathrm{~Hz}$. Other isotopes may be more problematic; for example, Potassium- $40\left({ }^{40} \mathrm{~K}\right)$ has a half life of $\sim 10^{9}$ yrs and natural fractional abundance of $10^{-4}$ [103], producing background events at $\sim 10 \mathrm{~Hz} / \mathrm{g}$. Table II provides details for isotopes relevant to the dielectrics listed for layer construction. For a discussion of other elements relevant to the rest of the experimental setup, see $[31,77,104]$.

Radioactivity can also be cosmic-ray induced. For example, high energy electrons in cosmic rays can produce radioactive ${ }^{14} \mathrm{C}$ from stable ${ }^{14} \mathrm{~N}$. If this must be avoided, the experiment may have to be run underground. Similarly, some layer synthesis processes employing high energy electrons or ions could result in accidental production of radioactive elements (e.g., ${ }^{14} \mathrm{~N}+e^{-} \rightarrow{ }^{14} \mathrm{C}$ from stray electrons in certain types of pulsed laser deposition).

It seems likely that some combination of small detector area, vetoing, and possibly purified materials and/or an underground laboratory, will allow us to discriminate signal photons from backgrounds in our setups. Similar backgrounds will affect other light DM experiments, and as prototypes of these are tested (e.g., [105]), we will gain more information on their properties.

\section{E. Sensitivity}

Figures 10 and 11 show the projected reach of our different experimental phases (Table I) for dark photon and axion DM. The mass range we consider is a well-motivated target for bosonic dark matter searches: dark photon dark matter is naturally produced by inflationary perturbations in the early universe, while for axions, a combination of inflationary perturbations and decays of nonperturbative defects can produce dark matter densities (Sec. V).

For the sensitivity plots, we assume that the dark photon or axion makes up all of the local DM density, $\rho_{\mathrm{DM}} \simeq$ $0.3 \mathrm{GeV} / \mathrm{cm}^{3}$. To obtain smooth coverage over an $e$-fold range in $\mathrm{DM}$ mass, we require $\sim Q$ half-wave stacks with quality factor $Q$, at fractional frequency spacings $\sim 1 / Q$. Equation (21) gives the converted power from the incoherent sum of these stacks. The sensitivity curves take an exposure time of $10^{6} \mathrm{~s}$ for each stack. For the 30-layer pathfinder configurations shown in Fig. 10, covering an $e$-fold mass range requires $\sim 150$ stacks; a 1 year total integration time would require running five stacks simultaneously. For the 100-layer (1000-layer) configurations in phases I and II, $400(\sim 4000)$ stacks can cover an $e$-fold reasonably smoothly. As discussed in Sec. II E, stacks at different frequencies can be run on top of each other, at the expense of a slightly spikier frequency profile. Figure 10 also shows an example of this "chirped stack" configuration, illustrating how a fractional frequency range of $\sim 30 \%$ can be covered by a single configuration.

In Figs. 10 and 11, we assume the most optimistic simultaneous-running case, such that a set of adjacent-infrequency stacks are aimed simultaneously at the same detector. This means that there is no dark count noise penalty from having the signal photons for a given DM mass coming from multiple stacks. If adjacent stacks have independent detector noise, then the coupling sensitivity will be degraded, though only by a factor of (noise counts) ${ }^{1 / 4}$.

The different curves within each phase of Figs. 10 and 11 represent different material pairs for the dielectric stacks. Sec. III A describes how a range of dielectric materials could be used to achieve close-to-optimal conversion rates over our entire range of frequencies, $\sim 50 \mathrm{meV}$ to $\sim 10 \mathrm{eV}$ (see Table II for examples). The lower end of this frequency range is limited by the threshold energy of low dark count single-photon detectors (Sec. III B; see Sec. VI for a brief discussion of bolometric detectors). The upper end is limited by the availability of simple dielectrics with low losses and high refractive indices at high frequencies, and is already well-constrained by other experiments and astrophysical observations.

Given the stringent constraints on the axion-photon coupling, Phase I parameters, with the addition of a $\sim 10$ Tesla magnetic field applied parallel to the layer surface, are necessary to improve on current bounds, Fig. 11. Phase II can significantly improve current sensitivities to axion dark matter, which couples to photons, in the mass range of $0.1-5 \mathrm{eV}$. The reach in coupling scales directly with applied B field, so large fields are required. However, we do not require a large volume: one stack can occupy as little as $(10 \mathrm{~cm})^{2} \times 10 \mu \mathrm{m}=0.1 \mathrm{~cm}^{3}$. Large magnetic fields with the above-mentioned volumes have been demonstrated [106] for both a resistive DC magnet (19 T, bore diameter $19 \mathrm{~cm}$ ) [107] as well as a superconducting magnet (21.1 T, bore diameter $10.5 \mathrm{~cm}$ ) [108]. The volume versus B field magnitude should be optimized to achieve the largest reach; in particular, if larger fields are available with smaller bore diameters, the area of an individual stack can be reduced and multiple stacks of smaller area can be run at the same time. To maximize the B field volume, mirror optics can be used to guide the light out of the magnet prior to focusing.

The axion-photon couplings that we could probe in Phases I and II are well below the KSVZ and DFSZ couplings for this mass range, respectively. However, for a generic QCD axion, the axion-nucleon couplings in this mass range are already constrained by the lack of SN1987A energy loss to axions [53]. We briefly discuss the possible extension of our experimental setups to frequencies $\lesssim 60 \mathrm{meV}$ in Sec. VI.

If a tentative signal were detected, the relevant mass range could be studied by increasing the $Q$ factor, either by placing an existing stack in a "cavity," or by creating stacks at the signal frequency with a larger number of layers (Sec. III C). Doing so increases the signal strength as well as giving finer frequency coverage, allowing for excellent discrimination from background, and, eventually, characterization of the signal properties. 
Figures 10 and 11 do not take into account the dark matter velocity distribution (similarly for the equations at the start of this section). As discussed in Sec. II, the effect of the DM velocity distribution, which has $\delta v \sim 10^{-3}$, is to spread out the converted power as a function of DM mass by a fractional mass range $\sim \delta v$, while preserving the massaveraged power. Consequently, for $N \ll 1000$ periods, the effect of the DM velocity distribution is expected to be small, while for $N \sim 1000$ periods, the effect on the power output from each individual stack can be $\mathcal{O}(1)$. For example, the total power output from a 500-period stack at a DM mass corresponding to its central frequency is only reduced by a factor $\gtrsim 0.8$ if the DM velocity distribution is set to the "standard halo model" [109] (SHM), as compared to zero velocity spread. ${ }^{7}$ For the 1000-period stacks assumed for Phase II, the peak power is reduced by $\gtrsim 0.5$ for the SHM, as compared to $\delta v=0$. However, since an experiment to cover a wide DM mass range would make use of many different stacks, with closely-spaced central frequencies, a wider DM velocity distribution results in almost the same number of overall converted photon events, but spread across different stacks. As discussed above, the effect of this on coupling sensitivity depends on whether multiple stacks are aimed at the same detector, in which case the sensitivity is as shown in Figs. 10 and 11. In the worst case of separate detectors, a reduction in peak power of $\sim 0.5$ results in a coupling sensitivity only a factor of $\sim 2^{1 / 4} \simeq 1.2$ worse.

\section{OTHER DARK MATTER CANDIDATES}

Spin-1 DM candidates, such as the dark photon, have an intrinsic polarization direction, so can convert to photons even in a locally isotropic target material. For spin-0 DM candidates, there must be some intrinsic direction to the target to determine the polarization of the converted photon, or else the conversion rate is suppressed by at least $v^{2}$, where $v$ is the DM velocity. In the case of an $a E \cdot B$ coupling, a background $B$ field provides this direction-however, for couplings to SM fermions, the target material itself must have some directionality to avoid $v^{2}$ suppression. ${ }^{8}$

Here, we give a brief overview of how dielectric haloscopes, using different target materials, can absorb some other DM candidates.

\section{A. $B-L$ vector}

Apart from the electromagnetic current, the other conserved current in the SM is $B-L$ (if neutrinos are Dirac).

\footnotetext{
${ }^{7}$ For a non-mirror-backed stack, the difference between the power emitted from each end can be significantly larger, as calculated in $[36,39]$.

${ }^{8}$ For axions with couplings to fermions, there is the additional feature that the target spins must be polarized to get appreciable coherent conversion.
}

This means that it could be consistently gauged, and that a new spin-1 particle coupled to $B-L$ could naturally be light without running into strong constraints from nonrenormalizable couplings. Phenomenologically, in situations where interactions with nuclei are subdominant (for example, refraction in a dielectric), a $B-L$ vector with coupling $\mathcal{L} \supset g_{B-L} X_{\mu} J_{B-L}^{\mu}$ will behave like a dark photon with $\kappa=g_{B-L} / e$. Consequently, our experiments will absorb $B-L \mathrm{DM}$ in the same way as they would dark photon DM.

A difference from the dark photon case is that the couplings of a $B-L$ vector to neutrons and neutrinos result in stronger constraints. The coupling to neutrons results in a fifth force between neutral matter [110], while a DM abundance with $m_{B-L} \gtrsim 0.1 \mathrm{eV}$ can decay to neutrinos, resulting in cosmological bounds [111,112]. These constraints mean that even our Phase II experiment could only just reach new parameter space.

\section{B. Scalar couplings}

New light scalar particles (i.e., with couplings to evenparity SM operators) can arise from UV physics in various ways, e.g., Higgs portal models [113], dilatons / radions [114-116], or moduli fields [117]. For low-energy interactions, the important couplings are those to EM via $\phi F F$, and fermion mass couplings $\phi \bar{f} f$ to the electron and nucleons.

The $\mathcal{L} \supset g_{\alpha} \phi F_{\mu \nu} F^{\mu \nu}$ coupling modifies the Maxwell equations to

$$
\begin{aligned}
\nabla \cdot E & =\rho+g_{\alpha} \nabla \phi \cdot E \\
\nabla \times B-\partial_{t} E & =J+g_{\alpha}(\nabla \phi \times B-\dot{\phi} E) .
\end{aligned}
$$

Compared to an axion-type $a F \tilde{F}$ coupling, the nonvelocitysuppressed $\dot{\phi}$ term now couples to the background electric field, rather than the magnetic field. A feasible magnetic field strength of $10 \mathrm{~T}$ corresponds, in natural units, to an electric field strength of $\sim 30 \mathrm{MV} / \mathrm{cm}$ - this is much larger than the electric field that can be applied to standard materials, and even in polar materials, the volume-averaged electric fields are $\lesssim \mathrm{MV} / \mathrm{cm}$. As a consequence, our experiments would give sensitivities weaker than current constraints from fifth force tests and stellar cooling [110,118].

The scalar couplings to fermion masses, $\mathcal{L} \supset g_{f} \phi \bar{f} f$, give a nonrelativistic interaction Hamiltonian (to first order in $g_{f}$ )

$$
H \supset g_{f} \phi+\frac{g_{f} \phi}{2 m_{f}^{2}}\left((\vec{p}-q \vec{A})^{2}-q \vec{\sigma} \cdot \vec{B}\right),
$$

where $q$ is the charge of $f, m_{f}$ is its mass, and $p$ and $\sigma$ are its momentum and spin operators. The first term gives rise to a force in the presence of a $\phi$ gradient, giving 
velocity-suppressed absorption rates. The second gives rise to an oscillating magnetic dipole moment, and to velocityand acceleration-dependent forces on the fermion. The coupling to electron mass, $\phi \bar{e} e$, and the couplings to nucleon masses, $\phi \bar{n} n$, are subject to strong constraints from fifth force tests and stellar cooling [41]. These mean that the velocity-suppressed absorption rate will not probe new parameter space. The other terms could lead to coherent absorption in directional target materials, but we leave such calculations to future work.

\section{DARK MATTER PRODUCTION MECHANISMS}

Light bosonic DM can be produced through a range of early-universe mechanisms, the simplest of which is purely gravitational production during inflation. For a spin-0 field, inflation "stretches" quantum fluctuations to super-horizon scales. After many $e$-folds of inflation, this results in the observable universe having, at the end, approximately the same background value of the field everywhere. This background value persists until the universe cools down enough that the Hubble rate is less than the mass of the particle, after which the scalar field starts oscillating, and behaves like matter.

In many models, axions have a periodic potential. For a field with a cosine potential, $V(a)=m_{a}^{2} f_{a}^{2} \cos \left(a / f_{a}\right)$, and a mass that does not change with temperature, its DM abundance today is [10]

$$
\frac{\Omega_{a}}{\Omega_{\mathrm{DM}}} \simeq\left(\frac{m_{a}}{\mathrm{eV}}\right)^{1 / 2}\left(\frac{f_{a}}{1.5 \times 10^{11} \mathrm{GeV}}\right)^{2}\left(\frac{\theta_{i}}{\pi / \sqrt{3}}\right)^{2},
$$

with $\theta_{i} \equiv a_{i} / f_{a}$, where $a_{i}$ is the post-inflationary value of the field over the observable universe. The scale $f_{a}$ is generally associated with the same physics that result in axion-SM couplings, which are then suppressed by $\sim 1 / f_{a}$. Hence, the mass and couplings ranges that our experiments will probe can naturally result in the correct DM abundance, through this "misalignment mechanism."

The QCD axion is slightly different from this case, both because its mass and symmetry-breaking scale are related, and also because its potential is temperature-dependent [32]. Consequently, misalignment production can only account for all of the DM if $m_{a} \lesssim 0.6 \mathrm{meV}$, well below the mass range we have been considering. However, there are post-inflationary production mechanisms that can increase this abundance. In particular, if the axion only becomes an effective degree of freedom post-inflation, then the phase transition in which this occurs will generically create a network of strings, whose cores contain the unbroken phase. This string network will evolve until around the QCD phase transition, when the temperaturedependent axion mass becomes comparable to the Hubble rate. At this point, it is expected to decay through the formation of domain walls.
Since the string network is expected to evolve towards an attractor solution, with eventual statistical properties almost independent of the post-phase-transition configuration, the axion DM abundance should theoretically depend only on the QCD axion mass. ${ }^{9}$ However, each of the stages involves complicated, nonequilibrium physics, and plausible predictions for the end abundance can differ by several orders of magnitude [119-123]. In theories with extra forms of new physics, there can also be other production mechanisms which give a full DM abundance of QCD axions at high masses, including the range we have considered [124-126]. These cosmological uncertainties motivate searching for QCD axion DM across as wide a mass range as possible.

Spin-1 DM can also be produced during inflation, through a process similar to the spin- 0 misalignment mechanism. Once again, quantum fluctuations are blown up by inflation to super-horizon scales. These do not start oscillating until the Hubble rate becomes smaller than the DM mass. The difference from the scalar case is that, during the time when the field is not oscillating, the magnitude of the vector field still decreases as $a^{-1}$, where $a$ is the FRW scale factor, and so the energy density in the field decreases as $a^{-2}$. In contrast, the potential energy density in a scalar field does not change until it starts oscillating. Consequently, the vector modes which are redshifted least are those which enter the horizon just as they are becoming non-relativistic. Larger-scale modes spent more time being redshifted before starting to oscillate, while smaller-scale modes spend time red-shifting rapidly as radiation. At late times, this results in a DM population that is dominated by modes at that special comoving scale, giving an overall density of [127]

$$
\frac{\Omega_{\mathrm{DP}}}{\Omega_{\mathrm{DM}}} \sim\left(\frac{m_{A}}{\mathrm{eV}}\right)^{1 / 2}\left(\frac{H_{I}}{5 \times 10^{12} \mathrm{GeV}}\right)^{2},
$$

where $H_{I}$ is the Hubble scale during inflation. Bounds on the CMB tensor to scalar ratio constrain $H_{I} \lesssim 10^{14} \mathrm{GeV}$ [128], so this production mechanism can produce all of the $\mathrm{DM}$ at masses $\gtrsim 10^{-5} \mathrm{eV}$.

\section{DISCUSSION}

In this work, we have outlined an experimental proposal for light bosonic dark matter searches in the $\sim \mathrm{eV}$ mass range. Using periodic, layered dielectrics along with modern low-dark-count photodetectors, these could have sensitivity to significant areas of new parameter space for dark photon and axion dark matter. Advantages of our proposal include simple target materials, small target

\footnotetext{
${ }^{9}$ This is true if the domain wall number is 1 ; if it is larger, than domain walls are long-lived, which can cause cosmological problems.
} 
volumes, and signal power emitted into collimated IR-UV photons, which can be efficiently detected.

The closest existing experimental proposals are the "dish antenna" searches $[129,130]$, which use a mirror to convert DM to collimated photons. Modeling a mirror as a very-high-refractive-index material, these are effectively a one-interface version of a dielectric haloscope, and consequently, only efficiently "use" a single-wavelength-high volume above the mirror. In contrast, dielectric haloscopes can achieve near-optimal dark matter absorption rates per unit volume (and, for axions, at a given background $B$ field), averaged over a range of possible dark matter frequencies. Achieving these high rates relies on constructing robust dielectric layers with high refractive index contrasts and large numbers of layers. At optical frequencies, it is relatively simple to operate in the regime where (thermal) backgrounds are small, so our fairly low $Q$ factors allow for broad mass coverage without sacrificing too much signal-to-noise.

The search for new light particles is ongoing on many fronts, including probes of new particles around the $\mathrm{eV}$ scale which are not the dark matter. Improved solar observations [118] and dark photon helioscopes could probe some of the same parameter space. The proposed IAXO axion helioscope experiment [131] and the ALPS II light shining through walls experiment [85] would improve on current axion-photon coupling bounds at masses $\lesssim 0.3 \mathrm{eV}$ and $\lesssim$ few $\times 10^{-4} \mathrm{eV}$, respectively. For axion couplings to fermions, the ARIADNE [132] experiment aims to search for new spin-dependent forces, and could probe axion-nucleon couplings for masses $\lesssim 10^{-2} \mathrm{eV}$. A signal found in a DM experiment would strongly motivate searches of these kinds, while conversely, signals in non-DM experiments could inform the dark matter search program.

The frequency coverage of our proposal is complementary to existing dark matter direct detection searches: at DM masses above $\sim 10 \mathrm{eV}$, absorptions have enough energy to ionize atoms in convenient target materials such as liquid xenon. Such detectors have the advantage of large target volumes, and easy detection of ionizations, enabling them to place strong constraints on dark photon DM [46, 133-136]. Direct detection experiments with lower thresholds are becoming possible, extending future reach toward the $\mathrm{eV}$ scale $[137,138]$. At DM masses $\lesssim \mathrm{eV}$, a number of other types of collective low-lying excitations have been proposed for DM absorption [31,129,136,139-142].

As reviewed in the previous section, the isotropic target materials we have considered are generally not optimal for detecting DM particles with different couplings. In recent work, [31] put forward a DM-to-photon conversion scheme based on a gas-phase target, which could probe a range of DM candidates and interactions through molecular excitations in the $\sim 0.2-20 \mathrm{eV}$ range.

A clear extension of our proposal is toward lower frequencies. The ultimate aim would be to close the sensitivity gap in axion-photon couplings between microwave proposals such as MADMAX, and the opticalfrequency experiments we have described. In particular, the best-motivated candidate for light bosonic dark matter is the QCD axion; the mass range which can give the full dark matter abundance is very broad given large theoretical uncertainties and different cosmological histories. Astrophysical constraints imply that the Peccei-Quinn (PQ) scale for a generic QCD axion is $\gtrsim 10^{8} \mathrm{GeV}$ [53], corresponding to masses $\lesssim 60 \mathrm{meV}$. Similarly, dark photon DM at lower frequencies is currently poorly constrained, and as discussed in Section V, could be produced during high-scale inflation.

Photon detection at these mid- to far-IR frequencies is more difficult than in the optical, but even with bolometric detectors, photonic haloscopes could reach interesting new parts of parameter space. For example, axion dark matter with the Kim-Shifman-Vainshtein-Zakharov (KSVZ) photon coupling would produce a signal power of $\sim 10^{-22} \mathrm{~W}$ (independent of $\mathrm{m}$ ) from a half-wave stack of 1000 highcontrast layers, with area $(10 \mathrm{~cm})^{2}$, in a 10 Tesla $B$ field. The same layers, in the absence of a $B$ field, would produce a signal power of $\sim 4 \times 10^{-12} \mathrm{~W}$ at meV energies, from dark photon $\mathrm{DM}$ with $\kappa=10^{-10}$ (which is the approximate astrophysical bound in the $\sim \mathrm{meV}$ range). For comparison, bolometers with noise equivalent powers of $\sim 10^{-20} \mathrm{~W} / \sqrt{\mathrm{Hz}}$ are achievable with current technology $[100,101]$. Blackbody radiation backgrounds become more important at these frequencies. If the layers are cooled to $T \ll m$, then most of the blackbody power is below the signal frequency and could be filtered out before reaching the detector. For example, only $\sim 10^{-12}$ of the emitted blackbody power from a stack at $4 \mathrm{~K}$ is at frequencies $>10 \mathrm{meV}$; for the parameters from above, this corresponds to $\sim 3 \times 10^{-25} \mathrm{~W}$. At smaller $m$, better than $\mathcal{O}(1)$ fractional frequency selectivity would be necessary. Other experimental challenges include materials, optics, layer construction, and background rejection. We leave detailed consideration of experiments at these frequencies to future work.

\section{ACKNOWLEDGMENTS}

We thank Nick Agladze, Francesco Arneodo, Karl Berggren, Aaron Chou, Adriano Di Giovanni, Savas Dimopoulos, Shanhui Fan, Andrew Geraci, Giorgio Gratta, Edward Hardy, Onur Hosten, Kent Irwin, Friederike Janusch, Axel Lindner, Ben Mazin, David Moore, Sae Woo Nam, Maxim Pospelov, Leslie Rosenberg, Gray Rybka, and Mark Sherwin for helpful discussions. We are grateful to Asimina Arvanitaki, Saptarshi Chaudhuri, Ken Van Tilburg and Xu Yi for helpful discussions and comments on the manuscript, and to Asimina Arvanitaki, Matthew Kleban, and Ken Van Tilburg for introductions to experimental colleagues and collaborators. We gratefully acknowledge the Kavli 
Institute for Theoretical Physics for hospitality during part of the project. This research was supported in part by the National Science Foundation under Grant No. NSF PHY1748958. M. B. is supported in part by the Banting
Postdoctoral Fellowship. Research at Perimeter Institute is supported by the Government of Canada through Industry Canada and by the Province of Ontario through the Ministry of Economic Development \& Innovation.
[1] V. C. Rubin and W. K. Ford, Jr, Astrophys. J. 159, 379 (1970).

[2] D. N. Spergel et al. (WMAP), Astrophys. J. Suppl. Ser. 148, 175 (2003).

[3] C. Yche, N. Palanque-Delabrouille, J. Baur, and H. du Mas des Bourboux, J. Cosmol. Astropart. Phys. 06 (2017) 047.

[4] V. Iri et al., Phys. Rev. D 96, 023522 (2017).

[5] A. Boyarsky, O. Ruchayskiy, and D. Iakubovskyi, J. Cosmol. Astropart. Phys. 03 (2009) 005.

[6] S. Weinberg, Phys. Rev. Lett. 40, 223 (1978).

[7] F. Wilczek, Phys. Rev. Lett. 40, 279 (1978).

[8] R. Peccei and H. R. Quinn, Phys. Rev. Lett. 38, 1440 (1977).

[9] B. Holdom, Phys. Lett. 166B, 196 (1986).

[10] I. G. Irastorza and J. Redondo, Prog. Part. Nucl. Phys. 102, 89 (2018).

[11] P. W. Graham, I. G. Irastorza, S. K. Lamoreaux, A. Lindner, and K. A. van Bibber, Annu. Rev. Nucl. Part. Sci. 65, 485 (2015).

[12] J. Jaeckel and A. Ringwald, Annu. Rev. Nucl. Part. Sci. 60, 405 (2010).

[13] P. Sikivie, Phys. Rev. Lett. 51, 1415 (1983); 52, 695(E) (1984).

[14] L. Krauss, J. Moody, F. Wilczek, and D. E. Morris, Phys. Rev. Lett. 55, 1797 (1985).

[15] P. Sikivie, Phys. Rev. D 32, 2988 (1985); 36, 974(E) (1987).

[16] S. J. Asztalos et al. (ADMX), Phys. Rev. Lett. 104, 041301 (2010).

[17] I. Stern, Proc. Sci., ICHEP2016 (2016) 198, [arXiv:1612 .08296].

[18] S. K. Lamoreaux, K. A. van Bibber, K. W. Lehnert, and G. Carosi, Phys. Rev. D 88, 035020 (2013).

[19] B. M. Brubaker et al., Phys. Rev. Lett. 118, 061302 (2017).

[20] Y. Kahn, B. R. Safdi, and J. Thaler, Phys. Rev. Lett. 117, 141801 (2016).

[21] S. Chaudhuri, P. W. Graham, K. Irwin, J. Mardon, S. Rajendran, and Y. Zhao, Phys. Rev. D 92, 075012 (2015).

[22] A. Caldwell, G. Dvali, B. Majorovits, A. Millar, G. Raffelt, J. Redondo, O. Reimann, F. Simon, and F. Steffen (MADMAX Working Group), Phys. Rev. Lett. 118, 091801 (2017).

[23] B. Phillips, in 2nd Workshop on Microwave Cavities and Detectors for Axion Research, Lawrence Livermore National Laboratory (2017), https://indico.fnal.gov/event/ 13068/timetable/\#all.detailed.

[24] J. Sloan, in 1st Workshop on Microwave Cavity Design for Axion detection, Livermore Valley Open Campus (2015), https://indico.fnal.gov/event/10138/timetable/\#all .detailed.
[25] P. Brun et al. (MADMAX interest Group), arXiv:1712.01062.

[26] A. J. Millar, G. G. Raffelt, J. Redondo, and F. D. Steffen, J. Cosmol. Astropart. Phys. 01 (2017) 061.

[27] J. D. Joannopoulos, S. G. Johnson, J. N. Winn, and R. D. Meade, Photonic Crystals: Molding the Flow of Light, 2nd ed. (Princeton University Press, Princeton, NJ, 2008).

[28] H. Sekoguchi, Y. Takahashi, T. Asano, and S. Noda, Opt. Express 22, 916 (2014).

[29] S. Noda, M. Fujita, and T. Asano, Nat. Photonics 1, 449 (2007).

[30] Z. L. Bushell, M. Florescu, and S. J. Sweeney, Phys. Rev. B 95, 235303 (2017).

[31] A. Arvanitaki, S. Dimopoulos, and K. Van Tilburg, arXiv: 1709.05354.

[32] G. G. di Cortona, E. Hardy, J. P. Vega, and G. Villadoro, J. High Energy Phys. 01 (2016) 034.

[33] F. Wilczek, Phys. Rev. Lett. 58, 1799 (1987).

[34] G. Rybka, A. Wagner, A. Brill, K. Ramos, R. Percival, and K. Patel, Phys. Rev. D 91, 011701 (2015).

[35] J. Jaeckel and J. Redondo, Phys. Rev. D 88, 115002 (2013).

[36] A. J. Millar, J. Redondo, and F. D. Steffen, J. Cosmol. Astropart. Phys. 10 (2017) 006.

[37] J. Jaeckel and J. Redondo, J. Cosmol. Astropart. Phys. 11 (2013) 016.

[38] J. Jaeckel and S. Knirck, J. Cosmol. Astropart. Phys. 01 (2016) 005.

[39] S. Knirck, A. J. Millar, C. A. J. O’Hare, J. Redondo, and F. D. Steffen, arXiv:1806.05927.

[40] H. An, M. Pospelov, and J. Pradler, Phys. Lett. B 725, 190 (2013).

[41] E. Hardy and R. Lasenby, J. High Energy Phys. 02 (2017) 033.

[42] J.H. Chang, R. Essig, and S. D. McDermott, J. High Energy Phys. 01 (2017) 107.

[43] P. W. Anderson, Philosophical Magazine Part B 52, 505 (1985).

[44] S. John, Phys. Rev. Lett. 58, 2486 (1987).

[45] H. An, M. Pospelov, and J. Pradler, Phys. Rev. Lett. 111, 041302 (2013).

[46] H. An, M. Pospelov, J. Pradler, and A. Ritz, Phys. Lett. B 747, 331 (2015).

[47] P. Gondolo and G. Raffelt, Phys. Rev. D 79, 107301 (2009).

[48] N. Vinyoles, A. Serenelli, F. L. Villante, S. Basu, J. Redondo, and J. Isern, J. Cosmol. Astropart. Phys. 10 (2015) 015. 
[49] V. Anastassopoulos et al. (CAST), Nat. Phys. 13, 584 (2017).

[50] H. Schlattl, A. Weiss, and G. Raffelt, Astropart. Phys. 10, 353 (1999).

[51] A. Ayala, I. Domnguez, M. Giannotti, A. Mirizzi, and O. Straniero, Phys. Rev. Lett. 113, 191302 (2014).

[52] D. Grin, G. Covone, J.-P. Kneib, M. Kamionkowski, A. Blain, and E. Jullo, Phys. Rev. D 75, 105018 (2007).

[53] J. H. Chang, R. Essig, and S. D. McDermott, arXiv:1803 .00993 .

[54] C. Patrignani et al. (Particle Data Group), Chin. Phys. C 40, 100001 (2016).

[55] L. Gao, F. Lemarchand, and M. Lequime, J. Eur. Opt. Soc.-Rapid publications 8, 13010 (2013).

[56] H. H. Li, J. Phys. Chem. Ref. Data 9, 561 (1980).

[57] Edmund Optics, The correct material for infrared (IR) applications, http://www.edmundoptics.com/.

[58] M. N. Polyanskiy, Refractive index database, https:// refractiveindex.info.

[59] F. Tran and P. Blaha, Phys. Rev. Lett. 102, 226401 (2009).

[60] T. Toyoda and M. Yabe, J. Phys. D 16, L97 (1983).

[61] LigenTec, silicon nitride (si3n4), https://www.ligentec .com/technology/.

[62] ISP Optics, IR optical materials, https://www.ispoptics .com/.

[63] T. Soma, J. Satoh, and H. Matsuo, Solid State Commun. 42, 889 (1982).

[64] G. Strauch, Isotope methods for dating old groundwater (International Atomic Energy Agency, Vienna, Austria, 2013).

[65] T. S. Moss, Proc. Phys. Soc. London Sect. B 63, 167 (1950).

[66] T. S. Moss, physica status solidi (b) 131, 415 (1985).

[67] J. M. Dudley, G. Genty, and S. Coen, Rev. Mod. Phys. 78, 1135 (2006).

[68] O. Butov, K. Golant, A. Tomashuk, M. van Stralen, and A. Breuls, Opt. Commun. 213, 301 (2002).

[69] W. Hermann and D. Wiechert, Mater. Res. Bull. 24, 1083 (1989).

[70] P. K. Bachmann, D. U. Wiechert, and T. P. M. Meeuwsen, J. Mat. Sci. 23, 2584 (1988).

[71] A. K. Sinha, H. J. Levinstein, and T. E. Smith, J. Appl. Physics 49, 2423 (1978).

[72] D. Hashimoto and K. Shimizu, Rev. Sci. Instrum. 79, 093102 (2008).

[73] O. Ziemann, J. Krauser, P. Zamzow, and W. Daum, POF Handbook: Optical Short Range Transmission Systems (Springer, Berlin Heidelberg, 2008).

[74] J. M, Optical Fiber Communications: Principles and Practice, Prentice-Hall international series in optoelectronics (Prentice Hall, Englewood Cliffs, NJ, 1992).

[75] S. Wang and P. Pirouz, Acta Mater. 55, 5500 (2007).

[76] K. Seshan, Handbook of Thin Film Deposition (William Andrew, Norwich, New York, 2012).

[77] K. Iga, IEEE J. Sel. Top. Quantum Electron. 6, 1201 (2000).

[78] Princeton Optronics, VCSEL diode laser, http://www .princetonoptronics.com/technology/.
[79] F. Zhang, B. Heinen, M. Wichmann, C. Möller, B. Kunert, A. Rahimi-Iman, W. Stolz, and M. Koch, Opt. Express 22, 12817 (2014).

[80] P. Russell, Science 299, 358 (2003).

[81] R. J. Tonucci, B. L. Justus, A. J. Campillo, and C. E. Ford, Science 258, 783 (1992).

[82] P. F. Moulton, J. Opt. Soc. Am. B 3, 125 (1986).

[83] S. Noda, in Optical Fiber Communication Conference (Optical Society of America, Anaheim, California, 2016) p. Th1K.1.

[84] S. Johnson and J. Joannopoulos, Photonic Crystals: The Road from Theory to Practice (Springer, New York, 2001).

[85] R. Bhre et al., J. Instrum. 8, T09001 (2013).

[86] Princeton Instruments, Industry-standard, high-performance ccd cameras, https://www.princetoninstruments.com/ products/PIXIS-CCD.

[87] A. Di Giovanni (private communications).

[88] F. Arneodo, M. Benabderrahmane, G. Bruno, V. Conicella, A. D. Giovanni, O. Fawwaz, M. Messina, A. Candela, and G. Franchi, Nucl. Instrum. Methods Phys. Res., Sect. A 893, 117 (2018).

[89] J. Dreyling-Eschweiler (ALPS-II), in Proceedings, 10th Patras Workshop on Axions, WIMPs and WISPs (AXIONWIMP 2014): Geneva, Switzerland, 2014 (2014), p. 63.

[90] B. Cabrera, R. M. Clarke, P. Colling, A. J. Miller, S. Nam, and R. W. Romani, Appl. Phys. Lett. 73, 735 (1998).

[91] B. S. Karasik, S. V. Pereverzev, A. Soibel, D. F. Santavicca, D. E. Prober, D. Olaya, and M. E. Gershenson, Appl. Phys. Lett. 101, 052601 (2012).

[92] A. E. Lita, A. J. Miller, and S. W. Nam, Opt. Express 16, 3032 (2008).

[93] N. Bastidon, D. Horns, and A. Lindner, arXiv:1509.02064.

[94] J. Dreyling-Eschweiler, N. Bastidon, B. Dbrich, D. Horns, F. Januschek, and A. Lindner, J. Mod. Opt. 62, 1132 (2015).

[95] K. Irwin and G. Hilton, Transition-edge sensors, in Cryogenic Particle Detection, edited by C. Enss (Springer, Berlin Heidelberg, 2005), p. 63.

[96] B. A. Mazin, in American Institute of Physics Conference Series, Vol. 1185, edited by B. Young, B. Cabrera, and A. Miller (American Institute of Physics, 2009), p. 135.

[97] P. Day, H. Leduc, B. A Mazin, A. Vayonakis, and J. Zmuidzinas, Nature (London) 425, 817 (2003).

[98] J. Gao, M. R. Vissers, M. O. Sandberg, F. C. S. da Silva, S. W. Nam, D. P. Pappas, D. S. Wisbey, E. C. Langman, S. R. Meeker, B. A. Mazin, H. G. Leduc, J. Zmuidzinas, and K. D. Irwin, Appl. Phys. Lett. 101, 142602 (2012).

[99] K. M. Rosfjord, J. K. W. Yang, E. A. Dauler, A. J. Kerman, V. Anant, B. M. Voronov, G. N. Gol'tsman, and K. K. Berggren, Opt. Express 14, 527 (2006).

[100] D. Farrah et al., arXiv:1709.02389.

[101] C. M. Bradford, P. F. Goldsmith, A. Bolatto, L. Armus, J. Bauer, P. Appleton, A. Cooray, C. Casey, D. Dale, B. Uzgil, J. Aguirre, J. D. Smith, K. Sheth, E. J. Murphy, C. McKenney, W. Holmes, M. Rizzo, E. Bergin, and G. Stacey, arXiv:1505.05551.

[102] P. Grieder, Cosmic Rays at Earth (Elsevier Science, New York, 2001). 
[103] M. R. Bhat, in Nuclear Data for Science and Technology, edited by S. M. Qaim (Springer, Berlin, Heidelberg, 1992), p. 817.

[104] C. Arpesella et al. (BOREXINO), Astropart. Phys. 18, 1 (2002).

[105] M. Crisler, R. Essig, J. Estrada, G. Fernandez, J. Tiffenberg, M. Sofo haro, T. Volansky, and T.-T. Yu (SENSEI), arXiv:1804.00088 [Phys. Rev. Lett. (to be published)].

[106] G. Apollinari, S. Prestemon, and A. V. Zlobin, Annu. Rev. Nucl. Part. Sci. 65, 355 (2015).

[107] Laboratoire National des Champs Magnetiques IntensesGrenoble, $19 \mathrm{~T}$ superconducting magnet, http://lncmi-g .grenoble.cnrs.fr/spip.php?rubrique105\&lang=en.

[108] National high magnetic field laboratory, 21 T superconducting magnet, https://nationalmaglab.org/user-facilities/nmrmri/nmr-instruments/900-mhz-ultra-wide-bore-magnet.

[109] C. McCabe, Phys. Rev. D 82, 023530 (2010).

[110] E. G. Adelberger, B. R. Heckel, and A. E. Nelson, Annu. Rev. Nucl. Part. Sci. 53, 77 (2003).

[111] Y. Gong and X. Chen, Phys. Rev. D 77, 103511 (2008).

[112] M.-Y. Wang and A. R. Zentner, Phys. Rev. D 82, 123507 (2010).

[113] F. Piazza and M. Pospelov, Phys. Rev. D 82, 043533 (2010).

[114] T. Damour and A. M. Polyakov, Nucl. Phys. B423, 532 (1994).

[115] T. R. Taylor and G. Veneziano, Phys. Lett. B 213, 450 (1988).

[116] N. Arkani-Hamed, L. J. Hall, D. Tucker-Smith, and N. Weiner, Phys. Rev. D 62, 105002 (2000).

[117] S. Dimopoulos and G. F. Giudice, Phys. Lett. B 379, 105 (1996).

[118] G. G. Raffelt, Stars as laboratories for fundamental physics (University of Chicago Press, Chicago, 1996).

[119] M. Gorghetto, E. Hardy, and G. Villadoro, arXiv:1806.04677.

[120] R. L. Davis, Phys. Lett. B 180, 225 (1986).

[121] C. Hagmann, S. Chang, and P. Sikivie, Phys. Rev. D 63, 125018 (2001).

[122] T. Hiramatsu, M. Kawasaki, T. Sekiguchi, M. Yamaguchi, and J. Yokoyama, Phys. Rev. D 83, 123531 (2011).

[123] V. B. Klaer and G. D. Moore, J. Cosmol. Astropart. Phys. 11 (2017) 049.
[124] M. Kawasaki, K. Saikawa, and T. Sekiguchi, Phys. Rev. D 91, 065014 (2015).

[125] A. Ringwald and K. Saikawa, Phys. Rev. D 93, 085031 (2016); 94, 049908 (2016).

[126] R. T. Co, L. J. Hall, and K. Harigaya, Phys. Rev. Lett. 120, 211602 (2018).

[127] P. W. Graham, J. Mardon, and S. Rajendran, Phys. Rev. D 93, 103520 (2016).

[128] P. A. R. Ade et al. (Planck), Astron. Astrophys. 594, A20 (2016).

[129] D. Horns, J. Jaeckel, A. Lindner, A. Lobanov, J. Redondo, and A. Ringwald, J. Cosmol. Astropart. Phys. 04 (2013) 016.

[130] J. Suzuki, T. Horie, Y. Inoue, and M. Minowa, J. Cosmol. Astropart. Phys. 09 (2015) 042.

[131] E. Armengaud et al., J. Instrum. 9, T05002 (2014).

[132] A. Arvanitaki and A. A. Geraci, Phys. Rev. Lett. 113, 161801 (2014).

[133] I. M. Bloch, R. Essig, K. Tobioka, T. Volansky, and T.-T. Yu, J. High Energy Phys. 06 (2017) 087.

[134] R. Agnese et al. (SuperCDMS), Phys. Rev. Lett. 116, 071301 (2016).

[135] E. Aprile et al. (XENON), Phys. Rev. D 94, 092001 (2016); 95, 059901(E) (2017).

[136] Y. Hochberg, T. Lin, and K. M. Zurek, Phys. Rev. D 95, 023013 (2017).

[137] J. R. T. de Mello Neto et al. (DAMIC), Proc. Sci., ICRC2015 (2016) 1221, [arXiv:1510.02126].

[138] J. Tiffenberg, M. Sofo-Haro, A. Drlica-Wagner, R. Essig, Y. Guardincerri, S. Holland, T. Volansky, and T.-T. Yu (SENSEI), Phys. Rev. Lett. 119, 131802 (2017).

[139] Y. Hochberg, T. Lin, and K. M. Zurek, Phys. Rev. D 94, 015019 (2016).

[140] Y. Hochberg, Y. Kahn, M. Lisanti, K. M. Zurek, A. G. Grushin, R. Ilan, S. M. Griffin, Z.-F. Liu, S. F. Weber, and J. B. Neaton, Phys. Rev. D 97, 015004 (2018).

[141] S. Knapen, T. Lin, M. Pyle, and K. M. Zurek, arXiv:1712.06598 [Phys. Rev. Lett. (to be published)].

[142] P. C. Bunting, G. Gratta, T. Melia, and S. Rajendran, Phys. Rev. D 95, 095001 (2017). 